\title{
TABLAS DE VIDA DE ESTADOS MÚLTIPLES: PERSPECTIVAS DE MOVIMIENTO Y TRANSICIÓN
}

\author{
JACQUES LEDENT * \\ International Institute for Applied Systems Analysis
}

\section{INTRODUCCIÓN}

ENTRE L OS diferentes tipos de modelos de la demografía matemática, uno que parece estar más claramente representado es aquel que se construye en base a varias extensiones de algunas de las ideas básicas sobre las que descansa el modelo de la tabla de vida. Modelos de este tipo constituyen un recurso útil para seguir las transiciones experimentadas, entre dos o más estados, en el tiempo y la edad, por un grupo de personas nacidas al mismo tiempo.

En el caso más simple —el de la tabla ordinaria de vida-hay dos estados: vida y muerte, y el énfasis se pone en la transición, no reversible, del primero al segundo. Una extensión directa es la tabla de vida de decrementos múltiples, que reconoce transiciones a más de un estado final absorbente (por ejemplo, varias causas de muerte). Sin embargo, en el caso de eventos renovables (como el matrimonio), este modelo no permite seguir personas que se han movido de un status a otro y analizar sus experiencias subsecuentes.

Tal problema puede resolverse con la ayuda de tablas combinadas que permitan tanto entradas (incrementos) como salidas (decrementos) de diferentes estados. Por su naturaleza general, estas tablas de vida de incrementos-decrementos son útiles para el análisis de status marital, participación en la actividad económica, paridades de nacimientos, migración interregional, etc. Las tablas de vida más simples de este tipo, llamadas tablas de vidajerárquicas de incrementos-decrcmentos, tratan el avance a través de estados sucesivos sin permitir reingresos. En versiones más complicadas, sin embargo, llamadas tablas de vida no jerárquicas de incrementos-decrementos, sí se permiten reingresos.

Existe una metodología bastante simple, y que ya está bien establecida, que subyace a la más simple de estas tablas de vida (ver Keyfitz, 1968), para la discusión del caso de la tabla ordinaria de vida y Jordán (1967) para la discusión del caso de decrementos múltiples. Esta metodología de tablas de vida jerárquicas de incrementos-decrementos fue expuesta por Hoem (1970a; 1970b) en el contexto del análisis de nupcialidad y fecundidad. Algunos de los temas relacionados con la elaboración de las tablas de vida no jerárquicas de incrementos-decremen-

\footnotetext{
* Quiero expresar mi agradecimiento al profesor Andrei Rogers, quien no sólo me instruyó acerca de las tablas de vida de incrementos-decrementos, sino también me proporcionó su generoso consejo y apoyo a lo largo del proceso de elaboración que llevó al presente artículo. También estoy en deuda con Nathan Keyfitz, Kenneth Land, Phil Rees, Robert Schoen y Frans Willekens por sus útiles comentarios a los primeros borradores y discusiones estimulantes referentes a las tablas de vida de incrementos-decrementos.
} 
tos fueron estudiados en el pasado (Depoid, 1938;Mertens, 1965; Jordán, 1967); sin embargo, no fue sino hasta fecha reciente que apareció en la literatura una discusión completa y sistemática de problemas metodológicos y empíricos suscitados con dicha elaboración (Rogers, 1973, 1975; Rogers y Ledent, 1975; 1976; Schoen y Nelson, 1974; Schoen, 1975; Schoen y Land, 1976, 1977; Krishnamoorthy, 1979).

El elemento clave para el desarrollo de las tablas de vida de incrementos-decrementos fue la verificación de que dichas tablas pueden ser vistas como generalizaciones de las tablas ordinarias de vida, en las que las funciones de las tablas de vida de estados múltiples en notación matricial se pueden sustituir por las funciones escalares de las tablas básicas de vida (Rogers y Ledent, 1975, 1976; Rogers, 1975). En la mayoría de los casos, la generalización matricial se volvió un asunto relativamente directo, pero no así en otros. El problema de la extensión matricial al caso de estados múltiples se basa en la estimación de matrices de probabilidades de supervivencia específicas por edad, en donde se originan todas las funciones de la tabla de vida de estados múltiples.

Para esta estimación, surgieron dos enfoques, que resultaron de las diferentes conceptualizaciones de "pasaje" entre distintos estados: el enfoque de la tabla ordinaria de vida, contempla "pasaje" como un evento instantáneo, similar al nacimiento o la muerte. Requiere datos de entrada en forma de tasas de ocurrencia/exposición. En contraste, el segundo considera "pasaje" como el resultado del cambio del estado de presencia de un individuo entre dos puntos en el tiempo y utiliza datos en forma de proporciones de supervivencia.

En resumen, varias contribuciones han llevado, en los últimos años, al desarro11 o de un tratamiento matemátic o formal, así como de métodos precisos de construcción, que le dan a las tablas de vida de incrementos-decrementos un status comparable con el de la tabla ordinaria de vida. El presente artículo revisa y resume la investigación realizada en las tablas de vida de incrementos-decrementos, a través de una exposición comprensiva, paralela a la exposición clásica de la tabla ordinaria de vida, como aquélla de Keyfitz (1968). Se da especial atención a los diversos métodos utilizados para su construcción, que en su etapa actual de desarrollo, aún presentan problemas tanto metodológicos como empíricos.

El artículo consta de dos partes principales. La primera, sección 2, expone la generalización de los conceptos de la tabla ordinaria de vida a las tablas de vida de incrementos-decrementos. También presenta una derivación teórica de la población estacionaria de estados múltiples y estudia diversos problemas relacionados con la conceptualización de las funciones de la tabla de vida de estados múltiples. La segunda, sección 3, discute explícitamente la construcción de las tablas de vida de incrementos-decrementos, y enfoca la estimación de matrices de probabilidades de transición específicas por edad. Primero se estudian y después se comparan las perspectivas de movimiento y transición.

La notación utilizada a lo largo de este artículo difiere de la que usaban los anteriores estudiosos de las tablas de vida de incrementos-decrementos. Iguala e intenta extender de una manera consistente la notación utilizada por Keyfitz (1968) para la tabla ordinaria de vida.

a) Las estadísticas relativas a la población, en la tabla de vida de estados múltiples, se denotan por letras minúsculas ( 
cepciones, tales como $\mathrm{L}_{\mathrm{x}} \mathrm{y} \mathrm{T}_{\mathrm{x}}$ ), mientras que las que se refieren a la población observada se denotan por mayúsculas.

b) La notación funcional $\mathrm{f}(\mathrm{y})$ se usa para representar una función de $y$ considerada como una variable continua, mientras que $f_{y}$ se usa cuando la función a la que uno se refiere es para un conjunto discreto de valores (y es un subíndice derecho).

En el caso de cantidades escalares, se han adoptado las siguientes reglas para explicar la existencia de estados en intercomunicación.

a) Los valores del estado específico de una estadística f se denotan por un sobreíndice derecho, específico de la región, por ejemplo, $\mathrm{f}^{*}$ o f*(y).

b) Los "pasajes" entre estados se denotan por sobreíndices colocados a ambos lados de la variable concerniente, por ejemplo, el sobreíndice izquierdo se refiere al estado de origen y el derecho, al estado de destino.

c) Si se necesita hacer referencia al estado de nacimiento o al estado de presencia a cualquier edad menor que la actual, se indica con dos subíndices izquierdos, que denotan, respectivamente, la región y la edad relevantes; por ejemplo, ${ }_{i y} \mathrm{f}^{\wedge}$ representa el valor de la función $\mathrm{f}$, característico de aque-

llos presentes a la edad $\mathrm{x}$ en el estado $\mathrm{j}$ que estaban en el estado $\mathrm{i}$ a la edad $y>y<\mathrm{x}$.

2. HACIA LA GENERALIZACIÓN DE LOS CONCEPTOS DE LA TABLA ORDINARIA DE VIDA

La tabla ordinaria de vida es un modelo de población estacionaria en el que un individuo nacido en un estado único (el de la vida) se mueve pronto o después de un tiempo hacia un estado absorbente (el de la muerte). La expresión de los factores de mortalidad en términos de probabilidad permite descubrir el número de individuos de una cohorte determinada que sobreviven a cualquier edad.

Algunas veces, sin embargo, el conocimiento de dicha cifra puede ser insuficiente y se desearía saber si los sobrevivientes están casados o divorciados, incorporados o no a la actividad económica, viviendo en la región 1 o en la región 2 , etc. Esto lleva a conocer una tabla de vida de incrementos-decrementos, en la que además del estado de la muerte, existen varios estados no absorbentes. Como la tabla de vida de decrementos múltiples, ésta es una tabla de vida de estados múltiples, pero difiere enormemente de la primera en que sí permite entradas hacia (incrementos) y salidas de (decrementos) los diferentes estados. Es un método que permite examinar la historia de mortalidad y movilidad de una cohorte de personas nacidas en uno o varios de los estados no absorbentes.

Es útil distinguir entre tablas de vida de incrementos-decrementos de rádix unitario - en las que la cohorte inicial está concentrada en un estado (como las tablas de vida de status marital y status ocupacional) — y las tablas de vida de incrementos-decrementos de rádixes múltiples —on las que la cohorte inicial está localizada entre varios o todos los estados en intercomunicación (como las tablas de vida multirregionales, esto es, tablas de vida de incrementos-decrementos apli- 
cadas a la migración interregional). De hecho, sin embargo, no existen diferencias reales fundamentales entre los dos tipos de tablas -esto se aclarará más adelante - $\mathrm{y}$ entonces el resto del presente artículo se aplica a ambos (excepto cuando se especifique de otra manera).

En esta sección, se intenta, principalmente, extender los conceptos de la tabla ordinaria de vida al caso de las tablas de vida de incrementos-de crementos, estableciendo un paralelismo con la metodología convencional de la tabla ordinaria de vida. Primero se presenta una derivación teórica de la población estacionaria de estados múltiples y después se discuten varios temas relacionados con la conceptualización de las funciones de las tablas de vida de estados múltiples.

\subsection{LA POBLACIÓN ESTACIONARIA DE ESTADOS MÚLTIPLES: UNA DERIVACIÓN TE Ó R I C A}

Para facilitar la comprensión, primero se presenta una revisión breve de las derivaciones principales de la tabla ordinaria de vida, seguida de las generalizaciones correspondientes de estados múltiples.

En la tabla básica de vida, el problema principal es estimar la curva de sobrevivientes, $1(y)$, a cualquier edad $y$, de una cohorte de $1_{0}$ bebés, por ejemplo. La estimación empieza con la definición de la tasa de mortalidad instantánea (o fuerza de mortalidad), $(x\{y)$, asociada con la edad .y. Esta definición aparece en la ecuación (1) del cuadro 1, en la que $\mathrm{d}(\mathrm{y})$ denota el número de muertes que ocurren en el grupo de sobrevivientes $1(\mathrm{y})$ entre las edades y y $y+\mathrm{dy}$, donde dy es pequeño.

Si se observa que la cohorte está cerrada, es decir, que no hay otra forma de salida más que la muerte, es fácil demostrar, en la ecuación (2), el decremento que ocurre a $1(\mathrm{y})$ entre $y$ y $y+$ dy. Entonces, sustituyendo la ecuación (2) en la (1), da la ecuación diferencial básica (3), que exprésala relación entre ji(y) y 1(y) (Keyfitz, 1968, pág. 5).

La solución integral de la ecuación (3) está dada en la (4), en la que Í2(y) se puede expresar como se muestra en la ecuación (5). Esto permite definir el número de sobrevivientes, $1_{x}$, a edades fijas $\mathrm{x}=0, \mathrm{n}, 2 \mathrm{n}, \ldots, \mathrm{z}$, aplicando - como se muestra en la ecuación (6) - un conjunto de probabilidades de supervivencia específicas por edad, $p_{x}$, definido por la ecuación (7). Nótese que tradicionalmente todos los intervalos de edad considerados son iguales en longitud, excepto el último, que es abierto: $\mathrm{z}$ años y más.

Ahora volvemos al caso de incrementos-decrementos, cuya presentación se comienza con la derivación de la población estacionaria de estados múltiples, estudiada por primera vez por Schoen y Land (1976) y Krishnamoorthy (1979).' La presente exposición de tal derivación está basada fuertemente en el trabajo de estos autores y, sin embargo, guiada por el deseo de aclarar la conexión entre la tabla ordinaria de vida y la de incrementos-decrementos.

I Una presentación un poco diferente, pero equivalente, de las tablas de incrementos-decrementos comienza con la especificación de las ecuaciones diferenciales hacia adelante de Kolmogorov de la cadena subyacente markoviana (Schoen y Land, 1977; Willekens, 1978). 
Supongamos que tenemos un sistema de estados no absorbentes (denotado por $\mathrm{i}=1, \ldots, \mathrm{r}) \mathrm{y}$ que la cohorte inicial está localizada entre $\mathrm{s}$ de ellos $(1<\mathrm{s}<\mathrm{r})$. Aquí, el problema principal es estimar las curvas de sobrevivientes por, estado específico, $1^{*}(\mathrm{y})$, a cada edad $y$. Consideremos a un individuo nacido en cualquier estado del sistema y presente en el estado i a la edadjy y examinemos su posible localización dy años después, donde dy es pequeño. Puede estar vivo en el mismo estado o en alguno de los otros no absorbentes o puede haber muerto en el intermedio. Ahora acortemos dy lo suficiente, tal que eventos múltiples - como un movimiento al estado j seguido de la muerte en este estado- puedan descartarse. Entonces, existen tres alternativas para un individuo considerado en un período corto de tiempo $\mathrm{dt}=\mathrm{dy}$ : no sufre evento alguno, es decir, permanecer en el estado i, y fallecer en el estado i o moverse a alguno de los restantes estados no absorbentes del sistema. En otras palabras, el "pasaje" de un estado a otro se considera como un evento instantáneo similar a la muerte en demografía convencional. Entonces, no hay ninguna diferencia fundamental entre una transferencia al estado $\mathrm{j}=1, \ldots$, $\mathrm{r}$ y una transferencia al estado de muerte, 5: por lo tanto, fallecer en el estado i se puede considerar como movimiento del estado $\mathrm{i}$ al estado de muerte, referido como estado $r+1$. Consecuentemente, se sigue que las tasas de mortalidad instantáneas y por grupos específicos, no son diferentes de las otras tasas de movilidad.

C U A D R O I

COMPARACIÓN TABULAR DE LA EXPOSICIÓN TEÓRICA DE LA TABLA ORDINARIA DE VIDA Y DE INCREMENTOS-DECREMENTOS

Tabla ordinaria de vida

Tabla de vida de incrementos-de crementos

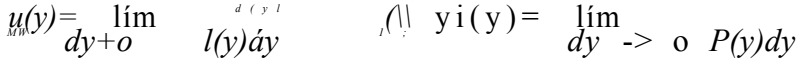

$$
\begin{aligned}
& l(y+\mathrm{O} »=l(y)-d(y) \quad \text { (2) } \%+\mathrm{dy})=t(y)-\stackrel{r}{2}^{+} * d l(y)+2 W(y)
\end{aligned}
$$

$$
/ \mathbf{O )}=\mathbf{i} \mathbf{2 O}) /(0)
$$

(4) $/(\mathbf{y})=$ Í2 (y) $1(0)$

Í2(y) = e x p M ( o d f j

(5) nO) $=\left[\mathrm{I}-\mathrm{Ai}_{\mathrm{AI}}(\mathrm{T},,) \mathrm{Ay},\right] \ldots\left[\mathrm{I}-\mathrm{u}\left(\mathrm{T}_{1}\right) \mathrm{Ay},\right]+\mathrm{e}^{\prime}(22)$

${ }^{\prime} x+n^{=} P j_{x}$

$(6)^{\mathrm{z} *},{ }_{+},=\mathbf{P A}$

(25) 
Sea que 'di(y) denote el número de movimientos del estado $\mathrm{i}(\mathrm{i}=1, \ldots, \mathrm{r}) \mathrm{a}$ cada estado $\mathrm{j}(\mathrm{j}=1, \ldots, \mathrm{r} 4-1, \mathrm{j} \wedge \mathrm{i})$ que hayan hecho, en un período corto de tiempo $\mathrm{dt}=\mathrm{dy}$, aquellos individuos que son miembros de un grupo de personas que sobreviven en el estado $i$ a la edad $j>, 1^{\wedge} y$ ). Como la exposición de estos individuos al riesgo de moverse antes de alcanzar y 4- dy es $l^{i}(\mathrm{y}) \mathrm{dy}$, el resultado es que $\mathrm{d}^{-} \mathrm{i}(\mathrm{y}) / \mathrm{l}^{\mathrm{i}}(\mathrm{y}) \mathrm{dy}$ es la tasa de movilidad correspondiente, del estado $\mathrm{i}$ al estado j (j i), asociada con la edad $y$. Entonces, podemos definir la tasa de movilidad instantánea (o fuerza de movilidad), $i i^{*}(y)$, como el valor determinativo de la tasa de movilidad, cuando dy $->0$.

$$
\mathrm{V}(\mathrm{y})=\mathrm{Hm} \quad \mathrm{Jfff} \quad, \quad 1=1 \ldots \mathrm{r}, /=1, \ldots, \mathrm{r}+1, j * L
$$

(Se supone que dicho límite existe para todos los estados del sistema y todas las $\operatorname{edades}^{\wedge}$.)

La definición (8) es realmente una generalización directa de la definición correspondiente (1) en la tabla ordinaria de vida. Nótese que, debido a los supuestos hechos, tenemos que

$$
\underset{\mathrm{y}=1}{\boldsymbol{r}+\mathrm{i}} \text { Wfy }</ \text { 'O), } \quad \mathrm{i}^{\prime \prime}=1, \ldots, \mathrm{r},
$$

o, equivalentemente, que

$$
\begin{aligned}
& \boldsymbol{r}+\mathrm{i} \\
& \mathrm{S} \quad V(y) \quad<-j \wedge \sim>\quad *=1 \\
& \mathbf{7}=\mathbf{1}
\end{aligned}
$$

En cuanto a los dos estados i y $\mathrm{k}$ - el último combinando todos los estados, excepto el i y el de muerte-, se puede establecer un esquema que una a las estadísticas d y 1 anteriormente mencionadas, como se muestra en el cuadro 2 . Es fácil entonces escribir la siguiente ecuación, indicando los decrementos e incrementos de los sobrevivientes, a la edadjy en la región i, de la cohorte inicial:

$$
f l(y-d y)=l(y)-" \mathrm{rf}^{\circ \prime}(y)-d^{*}(y) 4-{ }^{k} d(y \mid \quad i=1, \ldots, r .
$$

Recordando que $\mathrm{k}$ representa a todos los estados, excluyendo el i y el de muerte, y que el de muerte puede ser considerado como el $(r+1)$ ésimo estado del sistema, podemos re-escribir la ecuación (11) como sigue:

$$
/ /(\mathrm{y}+\mathrm{dy})=/^{i}(\mathrm{y})-^{\mathrm{r}} \mathrm{S}^{1} W(y)+\mathrm{Z} \quad i<f i(y \mid \quad /=1 \ldots \mathrm{r} \text {. }
$$


Estas ecuaciones forman la extensión de estados múltiples de la ecuación (2), que muestra el decremento en 1(y) en la tabla ordinaria de vida.

CUADRO 2

EVENTOS DEMOGRÁFICOS EN UN SISTEMA DE DOS REGIONES

Localización al

Localización al tiempo $t+y$

tiempo $\mathrm{t}=\mathrm{y}=\mathrm{dy}$

Presente en el presente en el

estado $\mathrm{i} \quad$ estado $\mathrm{k}$

\begin{tabular}{|c|c|c|c|}
\hline Vivo en el estado i & & ${ }^{k} d(y)$ & $\mu \leftrightarrow+d>0$ \\
\hline Vivo en el estado $\mathrm{k}$ & $' ; d^{*}(y)$ & & $l^{k}(y+a ́ y)$ \\
\hline Muerto & $d^{6} \quad(y)$ & ${ }^{k} d^{6}(y)$ & \\
\hline
\end{tabular}

Sustituyendo las ecuaciones (8) en las (12), lleva a (Schoen y Land, 1976):

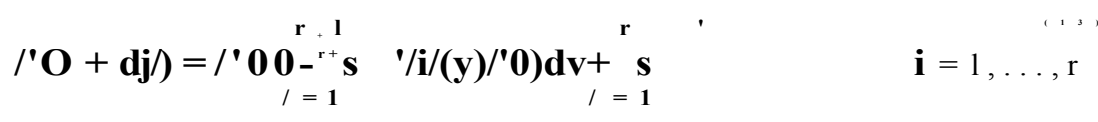

o más simplificadamente,

$$
l(y+d y)=l(y)-n(y) l(y) a ́ y,
$$

donde / (y) es un vector cuyo elemento típico es $\mathrm{P}(\mathrm{y})$ y

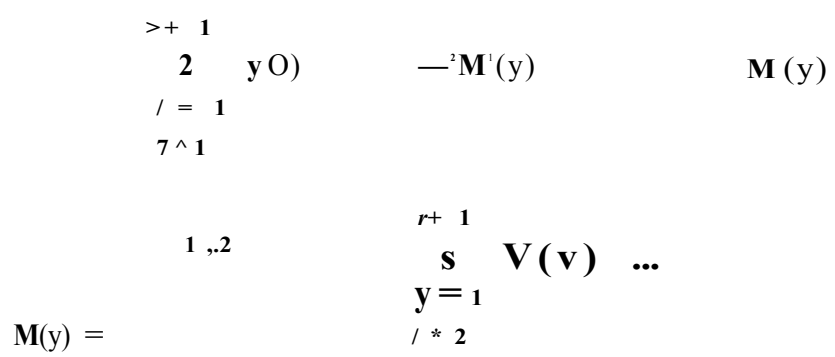

$\left.{ }^{1} \mathbf{N}^{\prime} \mathrm{O}\right)$ 
El i-ésimo elemento de la diagonal principal de ju(y) consiste en la fuerza total de movilidad de salida del estado i; el (i,j)ésimo elemento fuera de la diagonal contiene menos la fuerza de movilidad del estado j al estado i. Definiendo dl(y) como el vector diferencia $1(\mathrm{y}=\mathrm{dy})-1(\mathrm{y})$, lleva a re-escribir la ecuación (14) como sigue (Krishnamoorthy, 1979):

$$
\left.\left.\operatorname{Ir}_{\mathrm{r}} / \mathbf{C}\right)=-/ \mathrm{iC}\right) /(\mathrm{y})
$$

que aparece como una extensión directa de estados múltiples de la ecuación (3).

El sistema definido en la ecuación (16) admite r soluciones lineales independientes $1(\mathrm{y})_{k}(\mathrm{k}=1, \ldots, \mathrm{r})$, que, cuando se ponen lado a lado como las columnas de una matriz cuadrada, llevan a la matriz integral del sistema, $1(y)=[1(y)$ is ..., 1(y) r] (Gantmacher, 1959). Como cada columna de 1(y) satisface a la ecuación (16), la matriz integral1(y) satisface la ecuación.

$$
\wedge-H y)=-n(y V(y)
$$

Del teorema sobre la existencia y unicidad de la solución de un sistema de ecuaciones diferenciales lineales, se sigue que 1(y) está sólo determinada cuando se conoce el valor de 1(y) para algún valor inicial yo (Gantmacher, 1959):

$$
l(y)+{ }_{y o} f l(y) l(y o) .
$$

La matriz $\mathrm{yo}^{\wedge}(\mathrm{y})$, sólo definida como la solución normal para la ecuación (16), se llama matricante (Gantmacher, 1959). Su propiedad principal es la de transitivi dad:

$$
{ }_{n} n\left(y_{3}\right)={ }_{y_{2}} f l\left(y_{3}\right){ }_{y} \operatorname{Sl}\left(y_{3}\right) .
$$

En este caso, dejando que $\mathrm{y}_{0}=0 \mathrm{y}$ omitiendo el subíndice en ${ }_{0} \wedge(\mathrm{y})$, tenemos que

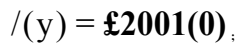

que es la extensión de estados múltiples de la ecuación (4).

Nótese que no se puede expresar a Í2(y) simplemente como una función de ju(y). La generalización directa de la ecuación (5) en

$$
\mathrm{I} 2(\mathrm{y})=\exp i^{y} / \mathbf{i}(\ll) \mathrm{dí}
$$

simplemente no se mantiene si ju(t) no es una matriz constante. Sin embargo, como han indicado Schoen y Land (1976) y Krishnamoorthy (1979), se puede de- 
terminar Í2(y) utilizando el cálculo infinitesimal de Volterra (ver Gantmacher, 1959, capítulo XIV):

$$
n(y)=\left[l-l i\left(T_{1}\right) \& y_{n}\right]\left[l-i x\left(r_{n}-y \mid y_{n_{-}}{ }_{1}\right] \ldots\left[\mathrm{I}-\mathrm{M}\left(\mathrm{r}_{1}\right) \mathrm{A} \mathrm{y}_{1}\right]+\mathrm{e},\right.
$$

donde e denota la suma de términos de orden mayor o igual a dos. Básicamente, el cálculo de $£ 2(y)$ a partir de la ecuación (22) requiere descomponer el intervalo básico $\left(0=\mathrm{y}_{\mathrm{o}}, \mathrm{y}=\mathrm{y}_{\mathrm{n}}\right)$ entre $\mathrm{n}$ partes, por la introducción de puntos intermedios

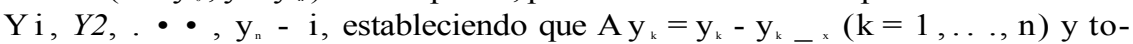
mando a $r_{k}$ como el punto intermedio del intervalo $\left(y_{k}{ }_{x}, y_{k}\right)$.

Habiendo derivado una matriz integral de la ecuación (16), necesitamos interpretarla. Primero supongamos que la cohorte inicial está localizada entre todos los estados $(s=r)$. Claramente $1(0)=1_{0}$ es una matriz diagonal que denota la localización de la cohorte inicial por estado específico: su elemento diagonal típico es $1 \mathrm{~J})$. Más aún, la i-ésima columna de 1(y) es el vector que represéntala localización de los sobrevivientes de $1 \mathrm{j}^{\wedge}$ por estado específico ala edadj $>$. De la ecuación (20), se sigue que su (j, i)ésimo elemento es el producto del (j,i)ésimo elemento ${ }_{i e} \boldsymbol{t} \boldsymbol{V}\{y)$ de $£ 2(\mathrm{y})$ con $1^{\wedge}$. Por lo tanto, ${ }_{i o} i 2 \mathrm{~J}(\mathrm{y})$ denota la probabilidad de que una persona nacida en el estado i sobreviva en el estado $\mathrm{j}$ a la edad $y$. Enresumen, (a) las $r$ soluciones independientes de la ecuación (16) son las r poblaciones estacionarias de estados múltiples que son generadas por cada rádix, es decir, cada una de las partes del estado específico de la cohorte inicial y (b)í2(y) es la matriz que muestra las probabilidades de supervivencia en el estado específico — a la edad $y$ - de los miembros de cada rádix.

Supongamos ahora que la cohorte inicial no está localizada entre todos los estados $(\mathrm{s}<\mathrm{r})$. En tales circunstancias, las columnas $\mathrm{r}-\mathrm{s}$ de $1(\mathrm{y})$ son simplemente vectores cero, pero esto sólo afecta ligeramente a la interpretación de $£ 2(y)$ : las columnas $\mathrm{r}$ - $\mathrm{s}$ de Í2(y) que corresponden a los estados vacíos inicialmente aparecen como las probabilidades hipotéticas de que prevalecerían si no hubieran estado vacíos inicialmente. Entonces, si $\mathrm{s}<\mathrm{r}$, la ecuación (16) aún admite $r$ soluciones independientes, pero sólo $s$ de ellas corresponden a poblaciones estacionarias multiestatales reales.

Nótese que Í2(y) es una matriz de probabilidad de transición propia: se puede demostrar que, como consecuencia de (a) las definiciones (8) relativas a las fuerzas instantáneas de movilidad y (b) las desigualdades (9) que las unen, todos los elementos de Í2(y) son no negativos y todos los elementos de cada columna suman un número menor o igual a uno. Tal propiedad se mantiene aún en casos menos favorables, es decir, cuando $\mathrm{s}<\mathrm{r}$. Por ejemplo, cuando el estado $\mathrm{k}$ está vacío inicialmente y no se ocupa por ningún individuo de edad menor a $\mathrm{y}_{\mathrm{a}}$, la k-ésima columna de Í2(y) para $\mathrm{y}<\mathrm{y}_{\mathrm{a}}$ no es un vector cero: como consecuencia de las definiciones relativas a $i i^{*}(y)$, su k-ésimo elemento es igual a uno. Se sigue que, $£ 2(y)$ nunca es una matriz singular y siempre tiene inversa.

Si recordamos nuestra interpretación del matricante, se sigue que la probabili$\operatorname{iad}^{\prime} \mathrm{pJ}_{\mathrm{x}}$ de que un individuo presente a la edad $\mathrm{x}$ en el estado i sobrevivirá en el astado $\mathrm{j}, \mathrm{n}$ años después es simplemente el ( $\mathrm{j}$, i)ésimo elemento de la matriz ${ }^{3} \mathrm{x} \sim \mathrm{x}^{\wedge}\left({ }^{\mathrm{x}}+{ }^{\mathrm{n}}\right)$ - Entonces, como consecuencia de la propiedad de transitividad

19) del matricante, tenemos que 


$$
\mathrm{p}_{x} \mathrm{i} 2(\mathrm{jc})=\mathrm{i} 2(*+\mathrm{rc})
$$

o, dado que $£ 2(\mathrm{x})$ tiene inversa,

$$
\mathrm{p}_{\mathrm{x}}=\mathbf{S} 2(\mathbf{J C}+\boldsymbol{n} \boldsymbol{W} \boldsymbol{x} \boldsymbol{J}
$$

una relación que es la análoga multiestatal de la ecuación (7). [Como con Í2(y) no es posible expresar $p_{x}$ como una función exacta de $/ x(y)$, tenemos que volver a utilizar el cálculo infinitesimal de Volterra.]

¿Podemos caracterizar más a $\mathrm{p}_{\mathrm{x}}$ ? Como $£ 2(\mathrm{y})$ es una matriz de probabilidades de transición propia, inmediatamente se obtiene, de la ecuación (24), que las matrices $\mathrm{p}_{\mathrm{x}}$ determinan un modelo de probabilidades de transición markoviano (Schoen y Land, 1976): Todas las matrices $p_{x}$ satisfacen las tres condiciones estándar especificadas en Cox y Miller (1965), o sea,

1) $0<<>/>\quad U=l, .-., n$

2) $0<\mathrm{S} \mathrm{H}<1 . \mathrm{i}=1, \ldots, \mathrm{r}$;

$$
/=\mathbf{i}
$$

3) la condición de transitividad, que es la consecuencia de la propiedad de transitividad (19), expuesta por el matricante de la ecuación (16).

Entonces, si $1_{x}$ denota el conjunto de matrices de $1(\mathrm{y})$ para $\mathrm{x}=0, \mathrm{n}, 2 \mathrm{n}, \ldots$, z, se obtiene la análoga de estados múltiples de la ecuación (6) como sigue:

$$
l_{x+n}=P_{x} l_{y}
$$

donde $\mathrm{p}_{\mathrm{x}}$ se obtiene de la ecuación (24).

De la exposición precedente, se puede contrastar la entrada en la ecuación (16) -que contiene r ecuaciones escalares que muestran cómo evolucionan sobre el tiempo los tamaños de cada grupo de sobrevivientes por estado específicocon el producto final [ecuación (20) o (24)] —que consiste de $r^{2}$ ecuaciones escalares que muestran los cambios en los estados de residencia de los individuos entre cada par de posibles orígenes y destinos. La razón para esta aparente ganancia de información se debe, por supuesto, a la introducción del supuesto markoviano cuando se definieron las tasas de movilidad instantáneas en la ecuación (8). Es precisamente la independencia de estas tasas de la historia de movilidad pasada de los individuos involucrados $\mathrm{y}$, por lo tanto, de su estado de nacimiento (en el caso de rádixes múltiples), lo que hace posible ver la evolución de la cohorte inicial como la evolución compuesta de r rádixes (reales o no) sujetos a los mismos patrones de movilidad y mortalidad por estado específico.

En resumen, el supuesto markoviano hace la extensión de los conceptos de la tabla ordinaria de vida al caso de incrementos-decrementos en forma relativamente directa: basta con la sustitución apropiada de los vectores y matrices por escalares. Esta extensión está ilustrada en el cuadro 1, que presenta una compara- 
ción tabular de la derivación de la población estacionaria en el caso ordinario y el de incrementos-decrementos.

\subsection{Funciones de la tabla de vida de estados múltiples}

Ahora volvemos a la tabla ordinaria de vida para revisar las definiciones de las funciones de la tabla de vida y después proseguir a su generalización de estados múltiples.

En la tabla ordinaria de vida, se piensa que 1(y) representa la evolución en el tiempo de una cohorte $1_{0}$, el número de años-persona vividos por los sobrevivientes de esta cohorte entre las edades x y x 4- n, $\mathrm{L}_{\mathrm{x}}$, se obtiene integrando 1(y) entre esas dos edades [ver ecuación (26) en el cuadro 3]. En forma similar, el número total de años que le restan por vivir a los sobrevivientes $1_{x}$ de $1_{0}, T_{x}$, se encuentra integrando $1(y)$ desde $x$ hasta infinito ${ }^{2}$ [ver ecuación (27)]. Entonces, para cada uno de los individuos $1_{x}$, la esperanza de vida promedio a la edad x está dada por la ecuación (29).

También se puede pensar la tabla ordinaria de vida como una distribución de individuos vivos en un tiempo dado (población estacionaria). En esta población, la tasa de mortalidad, $\mathrm{m}_{\mathrm{x}}$, relativa a los individuos de edades de $\mathrm{x}$ a $\mathrm{x} 4-\mathrm{n}$ es la razón entre el número de muertes, $\mathrm{d}_{\mathrm{x}}$, y la población expuesta, $\mathrm{L}_{\mathrm{x}}$. Como los cambios en el número de miembros de una cohorte sólo pueden ocurrir en forma de decrementos, debidos a la muerte, $\mathrm{m}_{x}$ se puede expresar simplemente como una función de las estadísticas 1 y L, como se demostró en la ecuación (30). Otra estadística interesante de una tabla de vida — necesaria para la evaluación numérica de los modelos de Leslie y Lotka- es la proporción de aquellos individuos en el grupo de edades de $\mathrm{x} a \mathrm{x}+\mathrm{n}$ que sobreviven al grupo de edades $\mathrm{de} x+\mathrm{n}$ a $\mathrm{x}+2 \mathrm{n}$. Se conoce como proporción de supervivencia y se define como la función de las estadísticas L [ver la ecuación (31)].

Existen dos generalizaciones diferentes de las funciones de la tabla básica de vida: una aparece como extensión vectorial; la otra como extensión matricial que subsume a la primera.

La primera generalización, introducida por Schoen y Nelson (1974) consta de las funciones de la tabla de vida de estados múltiples, que se derivan de las distribuciones $1 \mathrm{Hy}$ ) por edad por estado específico consideradas en su totalidad. Por ejemplo, Schoen y Nelson definen

$$
L_{x}^{i}=\int_{0}^{n} l^{i}(x+t) \mathrm{d} t
$$

2 La edad máxima a la que cualquier individuo puede vivir es infinito, ya que el último intervalo está abierto; nótese que en la práctica obtenemos $T_{x}$ a partir de:

$$
{ }^{T} x \underset{k}{\sim}{ }_{k}^{\wedge} x+k n
$$


como funciones que, como la variable $\mathrm{L}_{\mathrm{x}}$ de la tabla ordinaria de vida, tienen doble significado. Para una i dada, representa, primero, al número de personas vivas en el estado i entre las edades $\mathrm{x}$ y $\mathrm{x}+\mathrm{n}, \mathrm{y}$, después, al número de años-persona vividos por la cohorte inicial $1_{0}$ en el estado $i$ entre esas mismas edades. Las ecuaciones (32) se pueden re-escribir en forma vectorial, como sigue:

$$
L_{x}=\int_{0}^{n} l(x+t) \mathrm{d} t
$$

Similarmente, el número total de años que se puede esperar que vivan (en cada estado) los sobrevivientes de la cohorte inicial antes de morir se obtienen integrando ' $1(\mathrm{y})$ desde $\mathrm{x}$ hasta infinito:

$$
T_{x}=\int_{0}^{\infty} l(\mathrm{x}+t) \mathrm{d} t
$$

Con la idea de extender la definición (29) de esperanzas de vida a edades exactas, Schoen y Land (1976) definen la duración media de permanencia en el estado i a partir de la edad x para todos los sobrevivientes en el sistema a la edad $\mathrm{x}$, como el cociente del i-ésimo elemento de $\mathrm{T}_{\mathrm{x}}$ entre la suma de los elementos de $1_{x}$. Sin embargo, esta estadística no es suficientemente informativa y a uno le gustaría calcularla aún por el estado de presencia a la edad x. Desafortunadamente, no se puede hacer fácilmente, dado que se necesita distinguir la contribución a $\mathrm{T}_{\mathrm{x}}$, debida a los miembros de $1^{*}$, de aquéllas debidas a los miembros de $1_{i}(\mathrm{j}=$ $1, \ldots, n ; j \quad i)$. Entonces, necesitamos variables tales como $*_{x}$, que denoten el número de años que un miembro de $1^{\wedge}$ pueda esperar pasar en la región $\mathrm{j}$ antes de su muerte. Para esto, tenemos la siguiente ecuación que liga las estadísticas e, $1, \mathrm{~T}$ :

$$
/==! \quad x \quad x \quad T_{x}^{t}
$$

o más simplificadamente,

$$
e_{x} l_{x}=T_{x}
$$

donde el $(i, j)$ ésimo elemento de $e_{x}$ es ${ }^{\wedge} e_{x}$. 


\section{UA D R O 3}

GENERALIZACIÓN VECTORIAL DE LAS FUNCIONES DE LA TABLA

ORDINARIA DE VIDA

Tabla ordinaria de vida

$L_{x}=j j l(x+t) a ́ t$

$T_{x}=J \quad l(x+t) \dot{a} t$

Jo

$e x=\sim T \frac{}{/ \mathrm{v}}$

${ }^{\mathbf{M}} \boldsymbol{x}={ }^{\boldsymbol{k}_{x}}{ }^{{ }^{2} \boldsymbol{x}}$

$S_{x}+{ }^{L} *^{+} n$
Tabla de vida de incrementos-decrementos

$L_{x}=£^{\prime \prime} \quad l\{x+t) a ́ t$

$e_{x} l_{x}=T_{x}$

$\mathrm{TM}_{x} L_{x}=l_{x}-l_{x}+{ }_{n}$

$S_{x} L_{x}=L_{x+n}$

Evidentemente, la ecuación vectorial (36) es insuficiente para expresar $\mathrm{e}_{\mathrm{x}} \mathrm{de}$ la disponibilidad de $1_{x}$ y $T_{x}$. Sin embargo, sugiere que la generalización de $r$ distribuciones lineales independientes $1(y)$ hace posible que se obtenga $e_{x}$ (dado que $e_{x}$ es independiente de $1_{x}$ ). De hecho, como la ecuación diferencial (16), que subyace a la tabla de vida de incrementos-decrementos, admite r soluciones lineales independientes correspondientes a $\mathrm{r}$ rádixes (reales o no), basta con poner un subíndice adicional que se refiera al estado de nacimiento, para poder definir las funciones de la tabla de vida de estados múltiples que llevan a la derivación de $\mathrm{e}_{\mathrm{x}}$. Esta es la generalización matricial introducida por Rogers $(1973 ; 1975)$.

La segunda generalización de las funciones de la tabla ordinaria de vida empieza, entonces, con la definición de

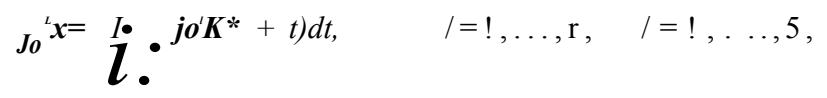

donde $j_{\varrho} l K^{x}+\mathbf{0}$ es el (i,j)ésimo elemento de $1(\mathrm{x}+\mathrm{t})$. Esta estadística representa el número de personas nacidas en $\mathrm{j}$ y vivas en el estado i de la tabla de vida entre las edades x y $\mathrm{x}+\mathrm{n}$-que es también el número de años*persona vividos en 


$$
\begin{aligned}
& \boldsymbol{L}_{x}=\boldsymbol{\ell}_{J o}^{\boldsymbol{n}} \boldsymbol{l}(x+t) d t \\
& \boldsymbol{T}_{x}=\boldsymbol{J} \quad \boldsymbol{l}(x+\mathrm{r}) \mathrm{df} \\
& \wedge 0 \\
& \boldsymbol{x}^{\wedge} \boldsymbol{x} \sim \boldsymbol{T}_{x} \\
& " x \boldsymbol{L}_{x}-(\mathrm{x} \sim \boldsymbol{l} \boldsymbol{x}+\boldsymbol{n} \\
& x^{\wedge} \boldsymbol{x} \sim \sim L_{x}+n
\end{aligned}
$$$$
\left.\mathrm{Lx}=\boldsymbol{f}_{J \mathbf{0}}^{n} \mathrm{~K}^{*}+t\right) \dot{a} t
$$$$
\mathrm{T}_{\mathrm{x}}-\boldsymbol{J}^{\mathrm{OO}} \boldsymbol{l}(x+t) d t
$$

0

$$
\begin{aligned}
& \mathrm{e}=\mathrm{T} 1 "{ }^{\prime} \\
& \mathrm{x}{ }^{\mathrm{A}} \mathrm{X}^{A} \mathrm{x} \\
& \left.{ }^{\mathrm{m}} \mathrm{x} \sim \mathrm{Ox}-1 \mathrm{x}+\mathrm{n}\right) \mathrm{L}_{\mathrm{x}}{ }^{\prime} \\
& x \sim \mathrm{L}_{\mathrm{x}}+{ }_{\mathrm{n}} \mathrm{L}_{*}
\end{aligned}
$$

el estado i entre esas mismas edades, por los miembros de la j-ésima rádix. Las ' ecuaciones (37) se pueden expresar más simplificadamente en la forma de una matriz de $\mathrm{r} \times \mathrm{r}$

$$
l(x+t) a ́ t
$$

El total de anos-persona vividos en el estado i para el grupo de personas nacidas en el estado $\mathrm{j} y$ actualmente a la edad $\mathrm{x}$ puede ser tomado como

$$
h \text { Tix }=\mathrm{CO}^{\mathrm{O} o H}{ }^{\prime}(x+t) d t, \quad i=1, \ldots, r, j=1, \ldots, s .
$$

Estos se pueden reunir en una matriz de r x r como sigue

$$
\underset{\text { Jo }}{\mathrm{T}_{\mathrm{v}}=/ 1(\mathrm{x}+t) \text { át. }}
$$

En la práctica, $T_{x}$ se puede obtener de

$$
\boldsymbol{T}=2 \text { i }
$$


En realidad, $L_{x}$ y $T_{x}$ tienen tantas columnas diferentes de cero como $1(x$ 4-1), es decir, $\mathrm{r}$ - s y, por lo tanto, la generalización matricial conlleva más información sólo en el caso de una tabla de vida de incrementos-decrementos de rádixes múltiples, es decir, cuando $\mathrm{s}>2$.

Ahora, con la adición de un subíndice que se refiere al lugar de nacimiento, podemos re-escribir la ecuación (35) de la siguiente manera:

$$
\underset{/-i}{\boldsymbol{I}} \mathbf{4} \boldsymbol{*}^{\wedge} \mathbf{0}^{\wedge}=\mathbf{0}^{\wedge} . \quad \quad »=1 . \quad . \quad k=l \ldots, s,
$$

o más simplificadamente,

$$
e_{x} l_{x}=T_{x}
$$

Por lo que, si $\mathrm{s}=\mathrm{r}$, tenemos que

$$
e_{x}=T_{x} l_{x},
$$

una relación que expresa la generalización matricial de la ecuación(29). La ecuación (44) define una matriz de esperanzas de vida por lugar de residencia a la edad $x$; sin embargo, también se puede definir una matriz de esperanzas de vida por lugar de nacimiento (ver Rogers, 1975).

Nótese que sustituyendo la ecuación (40) en la ecuación (44) y reemplazando $1(\mathrm{x}+\mathrm{t}) 1(\mathrm{x})^{\prime \prime} \operatorname{por}_{\mathrm{x}} 12(\mathrm{x} 4-1)$ da que

$$
e_{x}=j \quad S l(x+t) d t,
$$

una expresión que muestra la independencia de $\mathrm{e}_{\mathrm{x}}$ vis-à-vis el estado de localización de la cohorte inicial.

Ahora, si $\mathrm{s}<\mathrm{r}, 1_{\mathrm{x}}$ tiene $\mathrm{r}-\mathrm{s}$ columnas de ceros y no se puede invertir. Sin embargo, se puede superar esta dificultad de dos maneras alternativas. La primera es observar que, no importa si s es igual a r o no, la ecuación (45) se mantiene. Pero, en realidad, si en el cálculo se aplican tablas de vida de incrementos-decrementos, es difícil utilizar la ecuación (45). Por lo tanto es más factible extender la generalización matricial de las estadísticas $1, \mathrm{~L}$ y $\mathrm{T}$ de la tabla básica de vida. La idea es añadir a estas funciones un índice relacionado al estado de presencia a cualquier edad $y(0<\mathrm{y}<\mathrm{x})$ en vez de al estado de nacimiento (Ledent, 1978): esto lleva a definir estas funciones como ${ }_{y} 1_{x},{ }_{y} L_{x} y_{v} T_{x}$; por ejemplo, sea que y 1 , denote una matriz cuyo elemento típico,$l_{x}$ es el número de aquellos que, en el grupo de personas $1_{y}$ presentes a la edadjy en el estado $j$, sobreviven a la edad x en el estado i; las definiciones de $\mathrm{L}_{x} \mathrm{y}_{\mathrm{y}} \mathrm{T}_{\mathrm{x}}$ siguen inmediatamente de la de $1_{y} 1_{x}$. Entonces, tomando que $y=x$, obtenemos que 
[Sería necesario un truncamiento apropiado de las matrices involucradas en la ecuación (46) $\mathrm{si}_{x} 1_{\text {x }}$ tiene columnas cero y, por lo tanto, no admite inversa.] Más generalmente, tales funciones generalizadas $(\operatorname{con} \mathrm{y}=\mathrm{x})$ permiten la validez de la mayoría de las relaciones matriciales, derivadas previamente o por ser derivadas, cuando $\mathrm{s}<\mathrm{r}$.

Hasta aquí, nuestra discusión de las funciones de la tabla de vida de estados múltiples ha evolucionado alrededor de la generalización de las estadísticas 1, L y e de la tabla ordinaria de vida (para un resumen de esta discusión, véanse las primeras tres líneas de los cuadros 3 y 4 ).

Sin embargo, previendo la necesidad de aplicar el cálculo de las tablas de vida de incrementos-decrementos, nos gustaría discutir la generalización de otras dos estadísticas de la tabla ordinaria de vida: las tasas de mortalidad específicas por edad, $\mathrm{m}_{\mathrm{x}}$, $\mathrm{y}$ las proporciones de supervivencia, $\mathrm{s}_{\mathrm{x}}$, cuyas contrapartes multiestatales (en notación matricial) son la base para la implementación de los enfoques de movimiento y transición hacia la estimación de las probabilidades de transición específicas por edad.

Sea $\quad=£$ i) la tasa de movilidad específica por edad, análoga a la fuerza instantánea VHy)- Se puede definir simplemente con el número de decrementos $* \mathrm{~d}_{x}$, que ocurren en el estado $\mathrm{i}$ entre las edades x y x 4- $\mathrm{n}$ ( $\sin$ importar el estado de presencia a la edad $x$ de aquellos que se mueven de i a j), a la población expuesta $\mathrm{L}_{x}$ (Schoen y Nelson, 1974; Schoen, 1975):

$$
\mathbf{H}^{=}-\mathrm{T} \mathrm{T} \sim>\quad i=l^{\prime}{ }_{-}{ }_{9}, r, \quad /=1, \ldots, \mathrm{r}+1,
$$

De la definición (8) de la tasa de movilidad instantánea $\mathrm{V}(\mathrm{y})$, se sigue que el número de movimientos $* \mathrm{~d}_{\mathrm{x}}$ está dado por

$$
{ }^{\mathrm{r}} \mathrm{rf}=j j \quad W(x+t) V\{x+t) d t \quad /=!, \ldots, / \cdot, \quad /=!, \ldots ., \mathrm{H}-1, \quad j \pm i .
$$

Entonces, si recordamos la definición de $\mathrm{L}_{\mathrm{x}} \mathrm{y}$ la sustituimos en la (47), da que

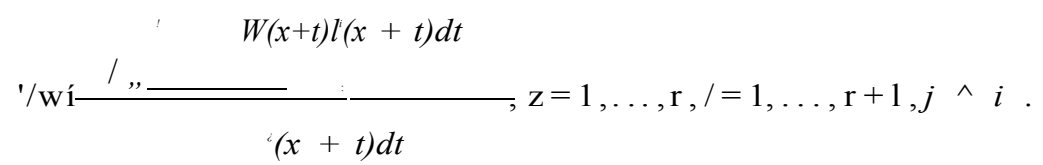

Notando que $1^{*}(\mathrm{x}+\mathrm{t})$ es una suma ponderada de las diversas probabilidades de supervivencia ${ }_{\mathrm{k}(\mathrm{)}} \mathrm{i}^{\mathrm{i}}(\mathrm{x}$ 4- t) (donde los pesos son los rádixes $1 £$ ), podemos reescribir la ecuación (49) como 


$$
. \mathbf{1}=1, \ldots \quad /=1, \ldots, \mathrm{H}-1, /
$$

una expresión que muestra que, en el caso de rádixes múltiples, el valor de ' $m_{x}$ está afectado por el estado de localización de la cohorte inicial, a menos que $\wedge(x+\mathrm{t})$ sea constante sobre el intervalo $(\mathrm{x}, \mathrm{x} 4-\mathrm{n})$.

Por lo tanto, en el caso de la tabla de vida de incrementos-decrementos de rádixes múltiples, las tasas de movilidad discretas, a diferencia de sus análogas instantáneas, no son en general independientes una de otra entre estados.

Ahora, ¿podemos establecer una relación, análoga a la ecuación de estado simple (30), expresando las tasas de movilidad en términos de las estadísticas 1 y L? La integración de las ecuaciones elementales de flujo (12) llevan a (Schoen y Nelson, 1974; Schoen, 1975):

$$
l_{x+n}=l_{*}^{\prime} \begin{array}{r}
r+1 \\
-2
\end{array} \quad \underset{7=1}{2} * d l \quad 1, \ldots, \mathrm{r} .
$$

Sustituyendo las ecuaciones defínicionales (47) en las ecuaciones (51), da entonces que

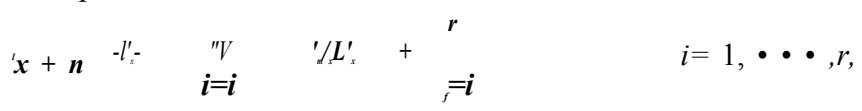

que se puede re-escribir como

$$
\text { 'x } \quad n=x \quad \sim x^{\prime} x
$$

donde $\mathrm{m}_{x}$ es la contraparte discreta de //(y) definida por la ecuación (15)

$$
\begin{aligned}
& \mathbf{2}^{r+1} m l \quad-2 m \boldsymbol{l} \text { ' } m: \\
& \text { 7. } \sim i \\
& 7^{\wedge} 1 \\
& -m l \\
& \mathbf{2}^{\mathbf{r}} m i \\
& 7 \sim 1 \\
& 7 * 2 \\
& -m i \\
& -{ }^{2} m l \\
& \mathbf{2}_{7=1}^{r+\mathrm{i}}
\end{aligned}
$$


Evidentemente, la ecuación vectorial (53) es insuficiente para obtener $\mathrm{m}_{x} \mathrm{de}$ la disponibilidad de $1_{x}, 1_{x}+{ }_{n} \mathrm{y} \mathrm{L}_{\mathrm{x}}$. Por lo tanto, es muy tentativo extender la ecuación (53) y escribirla en notación matricial

$$
1_{x+} \mathbf{n}^{=1} \mathbf{x}-m_{x} L_{x} .
$$

Sin embargo, tal relación no se mantiene siempre, ya que, como se dijo anteriormente, $\mathrm{m}_{\mathrm{x}}$ no es una matriz constante (depende de la asignación de los rádixes). Además, esto sugiere la constancia de las tasas de movilidad de la tabla de vida por lugar de nacimiento.

Así, la existencia de un patrón de movilidad predeterminado, como fue definido por las ecuaciones (8), no lleva a la constancia de las tasas de movilidad especificas por edad, sino a la constancia de las tasas que posteriormente serán referidas por el lugar de nacimiento. Por supuesto, en el caso de rádix unitario, no presenta ambigüedad alguna en las tasas de movilidad específicas por edad, dado que existe solamente un estado de nacimiento.

Ahora volvemos a la generalización en el caso de estados múltiples de las proporciones $\mathrm{s}_{\mathrm{x}}$ de supervivencia, que denotan la proporción de individuos de edades $\mathrm{x} a \mathrm{x}+\mathrm{n}$ que sobreviven a las edades $\mathrm{x}+\mathrm{n} \mathrm{ax}+2 \mathrm{n}, \mathrm{n}$ años después.

Sea que ${ }^{i} s_{x}$ denote la proporción de individuos presentes en el estado i entre las edades $\mathrm{x}$ y x 4- $\mathrm{n}$ que se mueven al estado $\mathrm{j}$ y sobreviven $\mathrm{n}$ años después en el estado de la población de edades $\mathrm{x}+\mathrm{n}$ a $\mathrm{x}+2 \mathrm{n}$. Entonces se demuestra fácilmente que la ecuación (31) se puede generalizar a

$$
{ }^{s} x^{\wedge} X \sim L_{x}+n \mathbf{J}
$$

donde $s_{x}$ es una matriz cuyo $(i, j)$ ésimo elemento es $s_{x}$.

Por supuesto que es tentativo extender la ecuación (56) y escribirla en notación matricial como

$$
{ }^{s} x^{L} x^{=}{ }^{L} x_{+} n^{\prime}
$$

De nuevo, tal extensión generalmente no se mantiene, debido a que los elementos $S_{x}$ dependen de la asignación de la rádix inicial. Para ver esto, escribamos $*_{S_{x}}$ como sigue

$$
\begin{gathered}
2 \quad k \ll i k o L_{x} n \\
k=1
\end{gathered}
$$

donde el numerador $\mathrm{k}^{\mathrm{a}} \mathrm{i} \mathrm{koL}_{\mathrm{x}+\mathrm{n}}$ representa la fracción del total de años vividos en el estado $\mathrm{j}$ entre las edades $\mathrm{x}+\mathrm{n} \mathrm{y} \mathrm{x}+2 \mathrm{n}$ por los individuos nacidos en $\mathrm{k}$ que también viven en el estado i entre las edades x y x 4- n. Recordando la notación iy $12 \mathrm{~J}\left(\mathrm{y}_{2}\right)$, se sigue que 


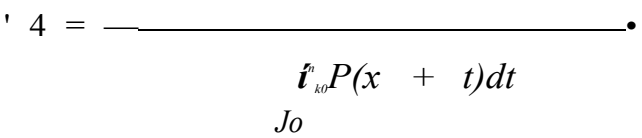

Sustituyendo la ecuación (59) en la (58), da, después de observar que ${ }^{\wedge} 1$ (x 4-1) es simplemente igual al producto $\mathrm{ko}^{\wedge i}\left({ }^{\mathrm{x}+} \mathrm{O}^{\wedge} \mathrm{O}{ }^{\prime} 1^{\mathrm{uc}}\right.$

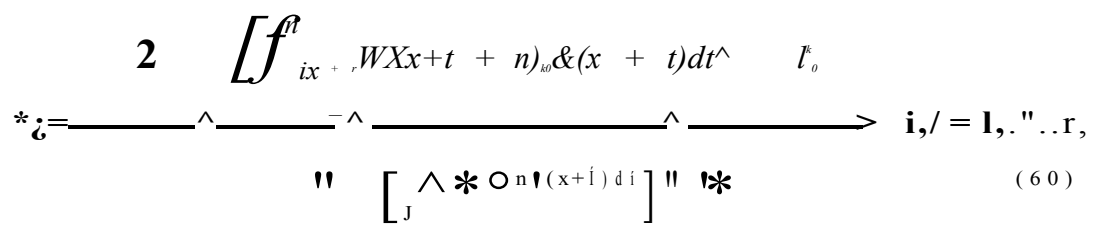

una expresión que demuestra que, en el caso de rádixes múltiples, el valor de ${ }^{i} \mathbf{S}_{x}$ está afectado por el estado de localización de la cohorte inicial. Además, esto sugiere la constancia de las proporciones de supervivencia en la tabla de vida por lugar de nacimiento.

Entonces, la existencia de un patrón de movilidad predeterminado, como fue definido por las ecuaciones (8), no lleva a la constancia de las proporciones de supervivencia específicas por edad, sino a la constancia de las tasas que posteriormente serán referidas por el lugar de nacimiento. Por supuesto, de nuevo no hay ambigüedad en el caso de rádix unitario, ya que existe solamente un estado de nacimiento.

En resumen, el elemento clave de esta generalización de la tabla ordinaria de vida reside en la definición (8) de las fuerzas instantáneas de movilidad: las propensiones instantáneas de un individuo a moverse son independientes de su historia de movilidad pasada. Esto lleva a un proceso markoviano para la interpretación de dichas tablas y garantiza la independencia, vis-á-vis la cohorte inicial, de las funciones de la tabla de vida de estados múltiples, características de una edad exacta. Las fórmulas (21), (24) y (45) muestran que funciones tales como £2(x), Px $\mathbf{Y}^{\circ} \mathbf{x}$ dependen sólo de las curvas ju(y) y bajo ninguna circunstancia están afectadas por el estado de localización de la cohorte inicial.

En contraste con estas funciones de la tabla de vida de estados múltiples relativas a edades exactas, aquellas funciones que se refieren a intervalos de edades, tales como, $\mathrm{m}_{\mathrm{x}} \mathrm{y}_{\mathrm{x}}$, están afectados por el estado de localización de la cohorte inicial (excepto en el caso de rádix unitario). Sin embargo, es tal el patrón de movilidad, que las funciones constantes de movilidad y proporciones de supervivencia originadas de cada rádix se pueden encontrar en la población estacionaria de estados múltiples.

De hecho, si se sigue el razonamiento con el que se llegó a los resultados anteriores, se obtienen resultados más generales, como que en cualquier tabla de vida, de incrementos-decrementos (de rádix unitario o de rádixes múltiples), las matrices de las tasas de movilidad, $\mathrm{m}_{\mathrm{x}}$, específicas por edad y las proporciones de su- 
pervivencia, $\mathbf{s}_{\mathrm{x}}$, dependen del estado de localización de la población estacionaria de estados múltiples a cualquier edad .y entre 0 y x.

\section{La aplicación del cálculo de las tablas de vida de incrementos-decrementos}

En la sección 2, se presentó una exposición teórica de las tablas de vida de incrementos-decrementos, poniendo énfasis en la importancia del supuesto markoviano introducido en la definición de las fuerzas instantáneas de movilidad. Pero, ¿en la práctica, cómo calculamos dichas tablas? El problema aquí es estimar las funciones de la tabla de vida de estados múltiples, especialmente las matrices de probabilidades de transición de las que todas las otras funciones se pueden generar.

Existen dos enfoques principales para dicha estimación, dependiendo del tipo de información que se tenga disponible: el enfoque de movimiento y el de transición (Ledent, 1978). El primero, que es semejante a la estimación clásica de la tabla ordinaria de vida, depende de la disponibilidad de tasas de ocurrencia/exposición. El segundo, que está relacionado con la estimación de la tabla ordinaria de vida a partir de información censal (Naciones Unidas, 1967), se basa en la información sobre proporciones de supervivencia. Rogers (1975) se refiere a estos enfoques como métodos de "opción 1" y "opción 2", respectivamente.

\subsection{Enfoque de movimiento y transición: sus características contrastadas}

Antes de regresar a la presentación de los dos enfoques de estimación, su esencia será caracterizada y comparada haciendo referencia al diagrama de Lexis.

El diagrama de Lexis de estado simple, que se encuentra en la literatura demográfica actuarial, se puede generalizar fácilmente para incluir el caso de estados múltiples (Rogers, 1973; 1975). Con el propósito de ilustrarlo, la gráfica 1 presenta el diagrama de Lexis de dos estados, que consta simplemente de dos diagramas de Lexis separados, uno directamente abajo del otro. Las líneas de vida de los migrantes entre las dos regiones unen a los dos diagramas. Nótense las tres clases de líneas de vida, representadas por A, B y C. La línea de vida A se refiere a un sobreviviente en el estado i que no migra. La línea $\mathrm{B}$ representa a un individuo en el estado i que emigra y sobrevive el intervalo unitario de edad en el estado j. La $\mathrm{C}$ se refiere a un individuo que se mueve del estado i al $\mathrm{j}$ y regresa antes del final del intervalo.

La primera posibilidad para conceptualizar el "pasaje" del estado i al estado j consiste en observar el sistema en un tiempo dado: entonces, se ve en la gráfica 1 que un individuo se mueve del estado $\mathrm{i}$ al $\mathrm{j}$ al tiempo $\mathrm{t}_{\mathrm{x}}$, otro al tiempo $\mathrm{t}_{\mathrm{z}} \mathrm{y}$ se observa también un movimiento del estado $\mathrm{j}$ al $\mathrm{i}$ al tiempo $\mathrm{t}_{3}$.

Una conceptualización alternativa del "pasaje" entre estados, se sigue de adoptar un tiempo de referencia basado en una observación del sistema en dos puntos en el tiempo más que en uno solo. Entonces, vemos en la gráfica 1 que el individuo correspondiente a la línea de vida B reside al tiempo t 4- 1 en un estado diferente de su estado de presencia al tiempo t; en contraste con el individuo representado por la línea $\mathrm{C}$ que vive en el mismo estado en ambos momentos, aunque haya tenido dos movimientos durante el intervalo de tiempo. 
En resumen, la primera conceptualización (del enfoque de movimiento) considera el "pasaje" o transferencia, como un evento (tal como el de muerte o nacimiento) que tiene lugar en un punto dado en el tiempo; en contraste con la otra conceptualización (del enfoque de transición) que considera dicha transferencia como el resultado de un cambio en el estado de presencia de un individuo entre dos puntos en el tiempo.

Es claro que el enfoque de transición conlleva menos información que el de movimiento, porque la simple observación de la localización de los individuos al principio y al final de un intervalo, más que a través de todo el intervalo, ignora

\section{G R Á F I C A I}

DIAGRAMA DE LEXIS DE DOS ESTADOS,

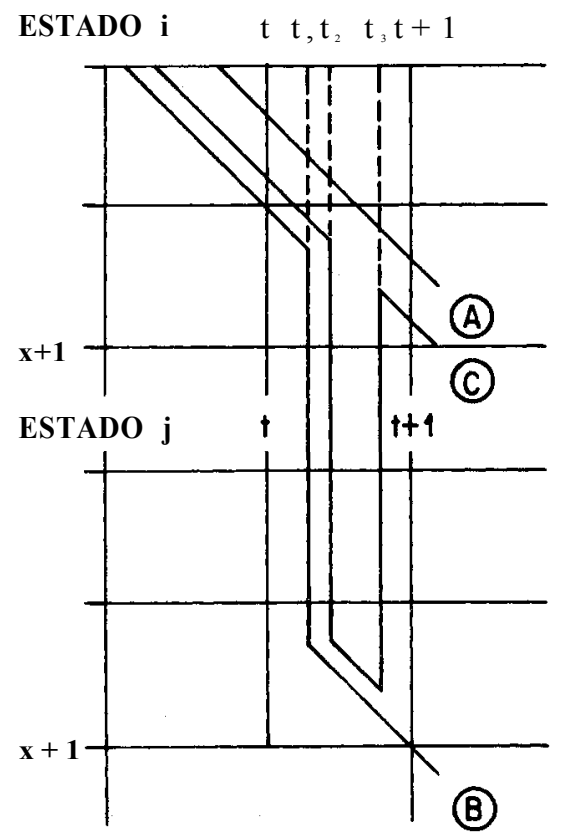

(F UENTE：ROGERS， 1973)

movimientos múltiples y migraciones de retorno dentro de un intervalo unitario de tiempo. Esto, sin embargo, no impide la confiabilidad del enfoque de transición vis-á-vis el de movimiento. El hecho es que cualquier modelo del tipo de una tabla de vida es un modelo de transición: es decir, en cualquier caso, se tienen que transformar los movimientos en transiciones. Esta transformación ocurre, ya sea dentro del modelo, cuando se estima $p_{x}$ (enfoque de movimiento) o fuera, cuando los datos originales vienen en forma de transiciones (enfoque de transición). 
Como se mencionó anteriormente, estos dos enfoques corresponden a los similares usados en el cálculo de las tablas ordinarias de vida. El de movimiento, como está enfocado a eventos que ocurren durante un intervalo de tiempo dado, permite calcular, de los datos originales, tasas de ocurrencia/exposición específicas por edad, análogas a la evaluación clásica de las tasas de mortalidad específicas por edad. Entonces, el problema es relacionar las matrices $p_{x}$ con las tasas de movilidad (mortalidad) observadas, en la misma forma que se relacionan las probabilidades de supervivencia con las tasas de mortalidad observadas en el caso de la tabla ordinaria de vida. En contraste, el enfoque alternativo que enfatiza las transiciones que ocurren a los individuos entre dos puntos en el tiempo, permite calcular para cada estado, proporciones de supervivencia específicas por edad (por estado de destino). El problema entonces es relacionar las matrices $p_{x}$ con dichas proporciones de supervivencia observadas, de la misma manera que se relacionan las probabilidades de supervivencia con las proporciones de supervivencia observadas en el caso de la tabla ordinaria de vida.

Nótese que en la mayoría de los casos en que se aplican las tablas de vida de incrementos-decrementos a situaciones reales, los datos vienen de una manera consistente con el enfoque de movimiento: éste es el caso tanto del análisis del status marital, como el de participación en la actividad económica — dos de los campos en los que más se aplican las tablas de vida de incrementos-de crementos (Schoen y Nelson, 1974; Schoen, 1975; Krishnamoorthy, 1979; Schoen y Land, 1977 —en el caso de status marital-; Hoem y Fong, 1976; Willekens, 1978 -en el caso de participación en la actividad económica).

Otro campo en el que se aplican las tablas de vida de incrementos-decrementos es el de migración interregional (Rogers, 1975). Aunque los datos originales pueden venir en forma de movimientos observados entre regiones sobre un período dado de tiempo (en naciones como Suecia u Holanda en que llevan registros de población, indicando los lugares de residencia de los individuos), también vienen de los censos de la población en forma de transiciones, es decir, número de personas de ciertas categorías que estaban en otra región específica uno o cinco años antes. Dichos datos de transiciones de migración han sido utilizados por Rogers (1975), Rees y WÜson (1977) y Ledent (1978).

De hecho, la estimación de una tabla de vida obtenida de las proporciones de supervivencia parece ser un método mucho más útil para el caso de estados múltiples (especialmente en el campo de migración interregional) que para el caso de estado simple, el que normalmente se usa sólo en caso de que falte información (para un resumen comparativo de las características de los enfoques de movimiento y transición para la construcción de tablas de vida de incrementosdecrementos, véase el cuadro 5).

En resumen, la implementación del enfoque de movimiento (transición) requiere ligar los patrones de movilidad de las poblaciones observadas a los de las estacionarias, que, como en la tabla ordinaria de vida, se hace estableciendo una simple igualdad de las tasas de movilidad (proporciones de supervivencia) con sus contrapartes observadas.

Recuérdese, sin embargo, que en la sección 2.2 obtuvimos el resultado de que en cualquier tabla de vida de incrementos-decrementos, las matrices de tasas de movilidad específicas por edad y las proporciones de supervivencia dependen del 
estado de localización de la población estacionaria de estados múltiples. En la estimación de una tabla de vida de incrementos-decrementos, es decir, la transición desde un modelo continuo, como se describió en la sección 2.1, a su equivalente discreto, surge un problema agregado. Aparentemente, la única solución a este problema es suponer que las tasas de movilidad específicas por edad (proporciones de supervivencia) son independientes del estado de localización de la población estacionaria de estados múltiples. Con este supuesto, la relación matricial (55) o (57), cualquiera que sea el caso, se mantiene y el procedimiento para el cálculo es, entonces, directo. De acuerdo con esto, tenemos que (Rogers y Ledent, 1976):

$$
m_{x}=\left(l_{x}-l_{x+n}\right) L_{x}^{\wedge}
$$

o (Rogers y-Ledent, 1975; Rogers, 1975)

$$
S_{x}=L_{x+} n L_{x}{ }^{-}>
$$

suponiendo que $\mathrm{L}_{\mathrm{x}} \mathrm{y}$, por lo tanto, $1_{x}$ tienen inversas (es necesario un truncamiento apropiado de las matrices involucradas en estas dos relaciones, $\mathrm{si}_{*} \mathbf{l}_{*}$ tiene columnas cero y, por lo tanto, no admite inversa). Más generalmente, aprovechando la ventaja de las funciones de la tabla de vida de estados múltiples por lugar de residencia a cualquier edad previa, tenemos que

$$
\text { rriy }=\left({ }_{x} l y-{ }_{x} l_{y}+{ }_{n}\right) L y
$$

o

$$
y \sim \sim{ }_{x} L y+n x L y \quad \mid
$$

Nótese que el supuesto que se hizo aquí para facilitar el cálculo de las tablas de vida de incrementos-decrementos es markoviano en el sentido de que las tasas de movilidad específicas por edad o las proporciones de supervivencia son hipotetizadas para ser independientes de la historia de movilidad de los sobrevivientes de la cohorte inicial. Sin embargo, este supuesto no es equivalente al markoviano introducido en el modelo teórico de la sección 2, cuando se definieron las fuerzas instantáneas de movilidad. ${ }^{3}$ Mientras que el segundo se refiere a características intrínsecas, el primero niega un papel al estado de localización de la población estacionaria y es verdaderamente un supuesto más fuerte.

Se concluye que para calcular una tabla de vida de incrementos-decrementos, ya sea por el enfoque de movimiento o el de transición, se requiere el uso de un supuesto subyacente, ligeramente diferente al que subyace al modelo teórico. Entonces, como se ve frecuentemente en la demografía matemática, el modelo discreto no se comporta como el modelo continuo paralelo.

Nótese, sin embargo, que si las tasas de movilidad observadas y las proporciones de supervivencia no son independientes del lugar de nacimiento, igualando 
las tasas de la tabla de vida con las observadas, daría estimaciones bastante imprecisas de las probabilidades de transición $\mathrm{p}_{\mathrm{x}} \mathrm{y}$ de las diversas funciones de la tabla de vida de estados múltiples. Por lo tanto, si se dispone de datos adecuados por lugar de nacimiento, es aconsejable calcular tablas de vida de incrementosdecrementos separadas para cada rádix. En el caso de la migración interregional, en el cual la dependencia de las propensiones de migración vis-á-vis el lugar de nacimiento (Long y Hansen, 1975), tal alternativa ha sido propuesta e implementada por Ledent (1980a) de la perspectiva de transición.

\section{U A D R O 5}

\section{CARACTERÍSTICAS DE LOS ENFOQUES DE MOVIMIENTO}

Y TRANSICIÓN CONTRASTADAS

Características

Conceptualización del "pasaje" entre estados
Enfoque de movimiento

Evento que tiene lugar en un tiempo dado, como en el caso de una muerte o un nacimiento.

\author{
Movimientos \\ I \\ Tasas de ocurrencia/ \\ exposición
}

Status marital

Participación en la actividad económica

Migración interregional (si los datos vienen de registros de la población)
Enfoque de transición

Cambio en el estado o presencia de un individuo entre dos puntos en el tiempo

Personas que se mueven (transiciones)

l

Proporciones de supervivencia

Migración interregional (si los datos vienen de la información de los censos)

3.2 Estimación obtenida de tasas de ocurrencialexposición: enfoque de movimiento

Como se hizo anteriormente, primero se presenta una revisión breve de la estimación correspondiente en la tabla ordinaria de vida y, después, la generalización a estados múltiples. El cálculo clásico de una tabla ordinaria de vida está basado en una relación de los patrones de mortalidad de las poblaciones observadas y de la tabla de vida, una liga que se establece más en un nivel discreto que en uno continuo y que usualmente involucra la igualdad de las tasas de mortali- 
dad específicas por edad en las poblaciones observadas y de la tabla de vida (denotadas por $\mathrm{M}_{\mathrm{x}} \mathrm{y} \mathrm{m}_{\mathrm{x}}$, respectivamente).

El método más simple para su construcción se sigue del supuesto de que $f i(y)$ es constante dentro de cada intervalo de edad $(x, x+n) y$, entonces, igual a $M_{x}$ (véase la ecuación (65) en el cuadro 6). En esas circunstancias, la sustitución de $M_{x}$ por $m_{x}$ en la ecuación (7) lleva a la probabilidad de supervivencia definida en la ecuación (66) en el cuadro 6. Esto permite determinar el conjunto de cantidades de supervivencia, $1_{x}$, aplicando la ecuación (6) y, después, el número de años-persona vividos, $\mathrm{L}_{x}$, de la ecuación (30), re-escrita como ecuación (67). En el caso del último grupo de edades, se puede obtener $L_{z}$ de la ecuación (67), suponiendo que $1=\ldots$ es igual a cero.

Un método alternativo para estimar la probabilidad de supervivencia, $\mathrm{p}_{\mathrm{x}}$, de las tasas de mortalidad observadas, $\mathrm{M}_{\mathrm{x}}$, se sigue del cálculo lineal de $\mathrm{L}_{\mathrm{x}}$, como se indicó en la ecuación (68); la fórmula correspondiente aparece como ecuación (69) en el cuadro 6.

En cualquier caso, no importa el método que se use, una vez que se han obtenido las cantidades L, se estiman las estadísticas $\mathrm{T}$, e y s de las ecuaciones (28), (29) y (31), respectivamente.

Estos dos métodos de construcción se pueden extender fácilmente al caso de incrementos-decrementos, debido a que las generalizaciones resultantes son consistentes con el requerimiento de que las tasas de movilidad específicas por edad sean independientes del estado de localización de la población estacionaria de estados múltiples.

El supuesto de las funciones de fuerza constante dentro de cada intervalo -sugeridas por Hoem (1970a; 1970b) y Hoem y Fong (1976)- llevan, como se dijo cuando se examinaron las fórmulas (50), a una matriz ambigua de tasas de movilidad. En tales circunstancias,

$$
\mathrm{ju}(\mathrm{j})=\mathrm{m}_{x}, \quad \operatorname{parax}<y<x+n,
$$

para que la igualdad de $m_{x}$ con su contraparte $M_{x}$ lleve a (Krishnamoorthy, 1979)

$$
\mathbf{P x}=\exp \left(-\ll \mathrm{M}_{\mathrm{x}}\right)
$$

Las fórmulas escalares pertenecientes al caso de dos estados aparecen como ecuaciones (72) a (76) en el cuadro 7. En la práctica, si r $>2$, se podría calcular Px de

$$
{ }_{\mathrm{p} x}=\mathrm{I}+\mathrm{n} \mathrm{M}_{\mathrm{x}}+-^{\wedge}-\mathrm{M}^{*}+\ldots .
$$

Una vez que se han obtenido las matrices de probabilidades de transición, de la fórmula (71), el conjunto de cantidades de supervivencia, $1_{\times}$, se obtiene fácilmente con la aplicación repetitiva de la ecuación (25).

En contraste, la estimación de los años-persona vividos, necesaria para calcular las otras funciones de la tabla de vida de estados múltiples, no es tan directa como en el caso de la tabla ordinaria de vida. El cálculo del número de años-perso- 
na vividos se sigue de la generalización matricial de la ecuación (53) a la (55), que ahora se mantiene. Pero, si ningún estado está vacío inicialmente, la ecuación (55) es insuficiente para generar todas las otras funciones.de la tabla de vida de estados múltiples. Entonces, podemos distinguir dos casos:

1) Si ningún estado está vacío inicialmente (es decir, si $s=r$ ), el número de años-persona vividos se puede calcular de la ecuación (55), re-escribiéndola como

$$
t_{x}=M_{x} \sim^{x}\left\{l_{x}-l_{x+n}\right)
$$

[Nótese que en el caso del último grupo de edades, la ecuación (78) sigue siendo válida si se pone que $1{ }_{z}{ }_{n}=0 ; y$, entonces,

$$
L_{z}=M \sim l,
$$

lo que ofrece una simple notación matricial de la solución escalar propuesta por Schoen (1975, apéndice).] En algunos casos, especialmente páralos grupos de edades más jóvenes, que exhiben pequeñas tasas de mortalidad, el determinante de $M_{x}$ se puede tomar en un valor cercano a cero $y$, entonces, el cálculo de su inversa puede resultar inexacto. Por esto, puede que se tenga que calcular $\mathrm{L}_{\mathrm{x}}$ de una fórmula alternativa que pudiera ser inconsistente con el conjunto de probabilidades $\mathrm{p}_{x}$.

Entonces, las estadísticas $\mathrm{T}$ y e se pueden estimar de las ecuaciones (41) y (44). Como para las probabilidades de supervivencia, sin importar si s es menor o igual a r, se debe, ya sea calcular dichas estadísticas por lugar de nacimiento (Ledent, 1978) u obtener valores aproximados, de la ecuación (62).

\section{U A D R O Ó}

MÉTODOS ALTERNATIVOS PARA CONSTRUIR TABLAS DE VIDA, ORDINARIAS

Y DE INCREMENTOS-DECREMENTOS

Método Tabla ordinaria de vida

Enfoque de movimiento (estimación obtenida de tasas de ocurrencia/exposición)

$$
\begin{aligned}
& m_{x}=M_{x} \\
& V(y)=\mathbf{T M}_{x} \quad \boldsymbol{y} \quad\left(P x=\operatorname{cxp}\left(-n M_{4}\right)\right. \\
& \mid x<y<x+n) \quad \mid \boldsymbol{L X}=
\end{aligned}
$$




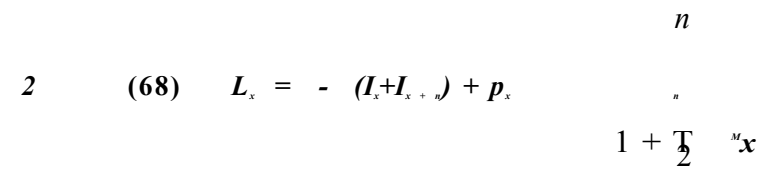

Enfoque de transición (estimación obtenida de proporciones de supervivencia)

$$
x \sim \$ x
$$

1

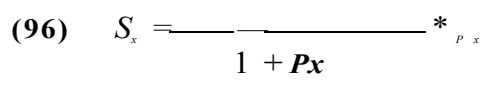

Tabla de vida de incrementos-decrementos

$$
\mathbf{M}(j)={ }^{\mathrm{m}} \mathbf{V} \quad \quad \quad \mathbf{P X}=\exp \left(-\operatorname{TI} \mathbf{M}_{\mathrm{x}}\right)
$$

(70)

$$
x<y<x+n \quad \mid \mathbf{L} \mathbf{x}=\mathbf{M}_{x}-^{-1}\left(\mathbf{l}_{x}-\mathbf{1}, \ldots,\right)
$$

$$
\mathbf{P} * \ll \mathbf{T}\left(-{ }^{s *}-»+\wedge\right)
$$

$\mathrm{p}_{\mathrm{x}}$ obtenida por interpolación entre las proporciones de supervivencia $S_{x}$ 


\section{U A D R O I}

EL CASO DE DOS ESTADOS EN EL ENFOQUE DE MOVIMIENTO

Supuesto exponencial

$\left(i(y)=m_{x}, \quad x<y<x+n\right.$

(70)

,$\left.\quad-\underline{\text { fo }}+{ }^{\prime} \boldsymbol{H}+{ }^{\prime} \wedge\right)$ e $\mathbf{x p}(\mathbf{r} \mathbf{w})-\left(\mathbf{r},+M_{r}+{ }^{\prime} \wedge\right)$ e $\mathbf{x} \mathbf{p}$ fo» $)$

$\wedge-n$

$' M_{x}^{\prime}\left[\exp \left(\mathbf{r}_{2} \mathbf{w}\right)-\exp \left(\mathbf{r}_{1} \ll\right)\right]$

$>2-'$ i

c $\quad f i-f i-r-\left(r_{2}+{ }^{\prime} M i^{\wedge} \operatorname{expí}^{\wedge}\right)+\left(\mathrm{r}_{x}+{ }^{\prime} \mathbf{M}^{\prime}\right) \exp \left(\mathrm{r}_{2} \mathrm{rt}\right)$

$\wedge 2=$

$$
r_{2}-r i
$$

donde

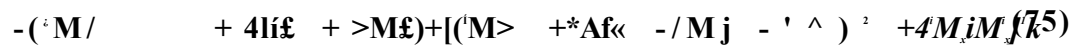

,$_{2=}-\left(\mathbf{M}^{\prime}+{ }^{i} \mathbf{M} \ll+{ }^{\prime} M_{x}^{\prime}+{ }^{\prime} M_{x}\right)-\left[\left(M_{x}+{ }^{\prime} \mathbf{M} \ll-{ }^{\prime} \mathbf{M j}->^{\prime} \mathbf{M}^{*}\right)^{2}+4 M_{x}^{\prime} M_{j}\right]^{\prime}{ }_{2}$

Supuesto lineal

$\left.\mathbf{L} \mathbf{c}=\wedge-\mathbf{C} *{ }^{+1} \mathbf{x}_{+} \gg\right)$

\footnotetext{
$t p t_{x}=\square-\longrightarrow \longrightarrow$

$l+{ }^{\wedge} M{ }^{*}+-y i M f_{x}\left(U i_{x} I V r\right)$

ip, = $\ll$ m i m- $s>$,
} 


$$
\begin{aligned}
& n M_{x}+\frac{{ }^{2}}{\sim} M_{x}\left(j M_{\mathrm{V}} M_{x} / \mathrm{J} \#{ }_{X}{ }_{X}\right) \\
& i_{n} h=f
\end{aligned}
$$

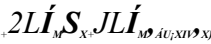

donde

$$
{ }^{r} L=1+\sim{ }^{M} x+
$$

Nota: ${ }^{*} \mathbf{q}^{\wedge}$ es la probabilidad de que una persona presente en el estado $i$ a la edad $x$ muera dentro de los siguientes $n$ años

F U E N T E: Schoen y Land (1977).

2) Si por lo menos uno de los estados está vacío inicialmente (es decir, si $\mathrm{s}<\mathrm{r}$ ), se debe recurrir a las funciones de la tabla de vida de estados múltiples, más adelante indicada por lugar de residencia a la edad $\mathrm{x}$, es decir, * 1 , $\mathrm{y}_{\mathrm{x}} \mathrm{L}$ y (ver sección 2.2). En este caso, el cálculo de diversas estadísticas de esperanzas de vida se obtiene utilizando la ecuación (63) en la que $M_{y}$ se sustituye por $\mathrm{m}_{y}$ :

$$
x^{\prime} y=M y^{\prime \prime \prime}\left(x^{\prime} y \sim x^{\prime} y_{+} n\right)>\quad y>x .
$$

Entonces, $e_{x}$ se obtiene de la ecuación (46), donde ${ }_{x} T_{x}$ se estima como $\mathrm{e}_{\mathrm{x}} \mathrm{L}_{\mathrm{y}}$.

$\mathrm{y}$

Existen muchas alternativas para este método, basadas en las funciones de fuerza constante, que también suponen que $\mathrm{m}_{x}$ es independiente del estado de localización de la población estacionaria. Fundamentalmente, dependen de la selección de un método explícito para calcular $\mathrm{L}_{\times}$. Para ver esto, nótese que, como consecuencia de la ecuación (55), tenemos que

$$
\left.\mathrm{a}-\mathrm{p}_{\mathrm{x}}\right) 1_{\mathrm{x}}=\mathrm{in}, \mathrm{L}_{\mathrm{s}}
$$

o, si $1_{x}$ tiene inversa,

$$
V_{x}=\left|-m_{x} L_{x}\right|_{x}-\mid
$$

ecuación idéntica a la que obtuvo Willekens (1978). Una posibilidades suponer un cálculo lineal de $\mathrm{L}_{\mathrm{x}}$ (Rogers, 1973; 1975): 
Combinando la ecuación (83) con la (61) lleva, después de sustituir $\mathrm{M}_{x}$ por $\mathrm{m}_{\mathrm{x}}$, a (Rogers y Ledent, 1976)

$$
\mathbf{p}^{*}=\left(-\mathbf{r}^{\wedge} \wedge-\mathbf{j}-\mathbf{M} \wedge 1\right.
$$

o, como las dos matrices I- $-M_{x}$ e I 4- $\mathrm{M}_{x}$ conmutan,

$$
p_{x}=\left({ }^{\mathrm{I}+} \mathbf{1 1}^{\mathrm{M} \boldsymbol{*}}\right)
$$

A diferencia de la fórmula (61), la (85) es válida, no importa si $\mathrm{s}<\mathrm{r}$. Las fórmulas escalares pertenecientes al caso de dos estados aparecen como ecuaciones (86) a (90) en el cuadro 7.

Nótese que, el cálculo lineal es involucrado de una manera menos restrictiva, al nivel de la generalización vectorial, es decir, si

$$
i^{*}=-\mathrm{f}-\left({ }^{\prime *}+\right.
$$

se obtiene que

$$
\left(\mathbf{r}_{+} \|=\mid \mathrm{i}-\mathrm{ir}{ }^{\mathrm{m}} \mathbf{x}\right)\left({ }^{\mathrm{i}+}-\mathrm{i}-{ }^{\mathrm{m}} * 1{ }^{2} *\right.
$$

combinando la ecuación (91) con la (61). Nótese también que, cuando consideramos una matriz del promedio de las duraciones de transferencia, que generaliza las $a_{x}$ de Chiang (1960) y de Rogers (1973; 1975), Ledent deriva una relación más general ligando $1_{\mathrm{x}}$ y $1_{\mathrm{x}+\mathrm{n}}$ que subsume la relación (92) del método de integración lineal.

Por lo tanto, se obtiene $\mathrm{p}_{\mathrm{x}}$ de la ecuación (85) sin importar si el cálculo lineal se hace al nivel de la generalización vectorial o matricial. De hecho, el supuesto de la integración lineal en forma vectorial [relación (91)] es suficiente para estimar $p_{x}$, pero es necesaria su extensión matricial [relación (83)] para calcular las otras funciones de estados múltiples.

Además, se puede arreglar la ecuación (85) para obtener

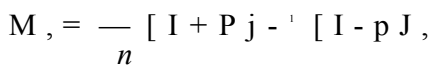

una relación que muestra que la hipótesis de que las tasas de movilidad específicas por edad son independientes del estado de localización de la cohorte inicial, está implícita en el supuesto de integración lineal.

A la fecha se han utilizado ambos métodos de construcción en el cálculo de las tablas de vida de incrementos-decrementos de rádix unitario. Hoem y Fong 
(1976) emplearon el supuesto exponencial (o fuerza constante de transición) en su análisis de participación de actividad económica y Krishnamoorthy (1979) y Schoen y Land (1977), en los análisis de status marital. El supuesto de integración lineal se usó tanto en un análisis de participación de actividad económica (Willekens, 1978) como en uno de los estudios citados de status marital (Schoen y Land, 1977). Nótese que en todos los casos se aplicaron métodos para edades individuales.

En contraste, el supuesto lineal ha sido usado para calcular tablas de vida multirregionales -tablas de vida de incrementos-decrementos de rádixes múltiples aplicadas a la migración interregional — para Finlandia (Manninen, 1979) y para otros países que llevan un sistema obligatorio de registro con referencia a grupos quinquenales de edad.

Un resultado curioso que se obtuvo en dicho estudio de Manninen para Finlandia es una probabilidad de retención negativa íp ${ }^{\wedge}$ para la región Uuden, una región de alta emigración. Tal resultado llama la atención al hecho de que las matrices de probabilidades $p_{x}$ en las ecuaciones (71) y (85) derivadas con la aplicación de los supuestos exponenciales y lineales, respectivamente, no son, a diferencia de las matrices de probabilidades $p_{x}$ que se obtuvieron en nuestra derivación teórica de la sección 2, matrices de probabilidades de transición propias. Se puede demostrar fácilmente que esto es válido en el caso de dos estados, utilizando las fórmulas expuestas en el cuadro 7. Es claro que, ya sea con el supuesto exponencial o lineal, (a) las probabilidades de migración y mortalidad pueden ser mayores de uno y (b) las probabilidades de retención pueden ser negativas. (Recuérdese que, en la tabla ordinaria de vida, $\mathrm{p}_{x}$ siempre está entre cero y uno en el caso exponencial, pero puede ser mayor que uno en el caso lineal $\mathrm{si}_{\mathrm{x}}>$ $2 / n$, es decir, si $M_{x}$ es grande $y / o n$ es grande.)

Por ejemplo, en un sistema de dos estados, se sigue, de las ecuaciones (72) y (86), respectivamente, que ${ }^{*} p_{\mathrm{x}}$ es negativo en el caso del supuesto exponencial, si

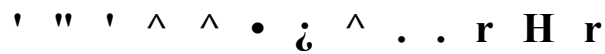

( 94 4)

y en el caso de integración lineal, si

$$
{ }_{x}^{\mathrm{M}}+{ }_{x}^{\mathrm{M}} \underset{x}{\mathrm{M}}-\underset{ }{y j}-
$$

Si se observan las desigualdades (94) y (95), se ve que, como se esperaba, es más fácil que ocurra una probabilidad de retención negativa mientras (a) mayor sea la tasa de mortalidad en el estado i o la tasa de movilidad del estado i al estado j y (b) mayor sea el valor del típico intervalo de edad n. En resumen, ${ }^{*} p_{x}$ puede ser negativo dado que las tasas discretas de movilidad no están limitadas: en teoría, podrían ocurrir constantemente movimientos en cualquier sentido en el sistema, así que las tasas de movilidad, estimadas sobre períodos de $\mathrm{n}$ años, pue- 
den ser demasiado grandes, ocasionando que las desigualdades (94) y (95) se mantengan. Esto muestra un gran contraste con el enfoque teórico, en el que las fuerzas instantáneas de mortalidad y movilidad están "restringidas" en la ecuación (10) ocasionando que las matrices $\mathrm{p}_{\mathrm{x}}$ sean probabilidades de transición propias.

En la práctica, si n se mantiene pequeño (por ejemplo, $n=1$ ), no ocurren probabilidades de transición impropias cuando se calcula una tabla de vida de incrementos-decrementos, porque la mayoría de las situaciones con las que nos enfrentamos involucran tasas de movilidad específicas por edad relativamente bajas. Sin embargo, aunque podemos obtener matrices $p_{x}$ con elementos entre cero y uno, la exactitud de los elementos $\mathrm{p}_{\mathrm{x}}$ que se obtienen de las ecuaciones (71) y (85) es cuestionable, especialmente para grupos de edad en los cuales las tasas de movilidad no son despreciables. La precisión de los estimados obtenidos disminuye cuando $\mathrm{n}$ aumenta.

En resumen, el cálculo de las tablas de vida de incrementos-decrementos, de las tasas de ocurrencia/exposición (enfoque de movimiento), se puede hacer generalizando los correspondientes métodos usados al caso de la tabla ordinaria de vida. Sin embargo, se ha demostrado que para que tal generalización sea posible, se requiere adoptar un supuesto que sea inconsistente con el apuntalamiento teórico de las tablas de vida de incrementos-decrementos descritas en la sección 2, o sea, la independencia de las tasas de movilidad específicas por edad del estado de localización de la población estacionaria de estados múltiples.

Dos de los métodos que más se utilizan están basados en los supuestos exponencial y lineal, que llevan a fórmulas que relacionan las probabilidades de transición con las tasas de movilidad, similares a las que se usan en los casos correspondientes de la tabla ordinaria de vida; la única diferencia es que las matrices reemplazan a los escalares.

Desde un punto de vista práctico, parece que en la evaluación numérica de las tablas de vida de incrementos-decrementos, de tasas de ocurrencia/exposición, surgen algunas preguntas sobre su exactitud con respecto a los datos específicos que se tienen a la mano. Cuando se hace una evaluación de este tipo, se debe poner especial atención a los valores de las diversas tasas de movilidad - especialmente cuando $\mathrm{n}$ es grande (por ejemplo, igual a cinco, como ocurre frecuentemente en el caso de datos sobre migración interregional)_-, así como para decidir la razonabilidad de los supuestos exponenciales y lineales.

\subsection{Estimación obtenida de tasas de supervivencia: enfoque de transición}

En algunas ocasiones, los datos sobre fenómenos de movilidad social vienen en forma de transiciones (personas que se mueven) en lugar de movimientos. Esto es común en el campo de migración interregional, donde los datos censales sobre cambios de residencia son comunes.

Supongamos que la información censal permite calcular las proporciones de supervivencia específicas por edad, resumidas en una matriz $\mathrm{S}_{x}$, la análoga observada de la tabla de vida $S_{x}$ definida en la sección 2.2. El problema para calcular $\mathrm{S}_{\mathrm{x}}$ se ha tratado en todas partes (ver Rogers y Von Rabenau (1971) para la estimación de $S_{x}$, de datos de migración de toda la vida, y Ledent (1978), de datos 
relativos a un período de tiempo fijo), por lo que aquí no se discute. Entonces, lo lógico es calcular una tabla de vida de incrementos-decrementos, generalizando el método algunas veces utilizado por demógrafos para calcular las tablas ordinarias de vida y que está basado únicamente en información censal. De acuerdo con este método (Naciones Unidas, 1967), como las probabilidades y las propensiones de supervivencia están relacionadas entre sí por la relación (96) en el cuadro 6 (si se adopta el método de integración lineal para calcular $\mathrm{L}_{\mathrm{x}}$ ), es posible estimar un conjunto de $\mathrm{p}_{\mathrm{x}}$ considerando las proporciones de supervivencia $\mathrm{S}_{\mathrm{x}}$ iguales a sus contrapartes observadas.

En teoría, este método se puede generalizar al caso de estados múltiples, en el que las matrices aproximadas de proporción de supervivencia $\mathrm{S}_{\mathrm{x}}$ están relacionadas con las matrices de probabilidad de supervivencia $p_{x}$ por la análoga matricial de la ecuación (96) (Rogers, 1975):

$$
r
$$

Sin embargo, como apuntó Ledent (1978), este procedimiento es mucho menos efectivo aquí en el caso de la tabla ordinaria de vida. Esto se debe, principalmente, a que las proporciones de supervivencia observadas reflejan la consolidación de los movimientos migratorios cuyo patrón puede haber variado a través del período observado. Entonces, las probabilidades de supervivencia que se observaron por tal método son valores promedio, y es más factible que sean muy imprecisos, debido al método particular de promedios implícito en la ecuación (97). Esto contrasta con el caso de la tabla ordinaria de vida, donde siempre que las proporciones de supervivencia hayan sido observadas correctamente, las tasas promedio de mortalidad implícitas en la ecuación (96) reflejan promedios aceptables (dado que los patrones de mortalidad cambian mucho menos que los patrones de migración). Además, debido a que el procedimiento para la estimación está basado en una fórmula que relaciona las estadísticas de dos grupos consecutivos, los errores hechos en la estimación de un grupo dado de edad pasan al siguiente: el "ruido" introducido, entonces, puede pasar a todo el conjunto de grupos de edades al que se aplique este procedimiento.

Por esta razón, Rees y Wilson (1977) por el contrario estiman el conjunto de probabilidades de supervivencia a partir de la siguiente aproximación:

$$
\mathrm{Px} \sim \mathrm{T}\left({ }^{\mathrm{s}} \mathbf{X}-\ll+{ }^{+s} \mathbf{X}\right) \text {. }
$$

[Nótese que, alternativamente, se podría pensar en estimar $\mathrm{p}_{\mathrm{x}}$ como la media geométrica (en lugar de aritmética) de las dos matrices de las proporciones de superviviencia consecutivas $\mathrm{S}_{x-{ }_{n}}$ y $\mathrm{S}_{\mathrm{x}}$.] Para el primer grupo de edades, se debe modificar la fórmula: sea que $s_{-^{n}}$ denote la probabilidad

de que los recién nacidos sobrevivan al final del intervalo (Ledent, 1978); entonces, se puede estimar $\mathrm{p}_{0}$ de 
Esto muestra que por más imperfecto que parezca el método definido en la ecuación (98), proporciona mejores resultados que los que se obtienen con el procedimiento basado en la ecuación (97), especialmente en el caso de grupos de edades avanzadas.

En vista de esto, propuse (Ledent, 1980b) un método de estimación que es mejor que el sugerido por Rees y Wilson (1977). Este método mejorado empieza mostrando que $S_{x}$ es, de hecho, un promedio ponderado de las probabilidades de supervivencia $\mathrm{p}_{\mathrm{x}+\mathrm{k}_{\mathrm{n}}}$, donde $\mathrm{k}$ toma todos los valores entre cero y uno. Entonces, se sugiere que una estimación adecuada de $\mathrm{p}_{\mathrm{x}}$ se puede obtener interpolando entre las proporciones de supervivencia observadas $s_{x}$, pero de una manera menos imperfecta que en la aproximación (98). Para cada par de estados no absorbentes i y j, tal interpolación se puede hacer fácilmente usando funciones spline cúbicas que se usan cada vez más en el campo de la demografía (McNeil et al, 1977). La ordenada de las curvas continuas obtenida así representa la probabilidad de que un individuo presente a la edad.y en el estado i esté presente en el estado $\mathrm{j}, \mathrm{n}$ años después. Las probabilidades requeridas se pueden encontrar como los estimadores de estas curvas a edades $x=0, n, 2 n, \ldots, z$.

Una ventaja de este procedimiento de interpolación con respecto a los métodos basados en las ecuaciones (97) y (98) es que no requiere necesariamente de proporciones de supervivencia calculadas para un período $\mathrm{T}$ igual a la longitud del grupo típico de edades, $n$. Si t $n$, el procedimiento de interpolación también permite estimar un conjunto de probabilidades de transición $p_{x}$ para $x=0$, $\mathrm{T}, 2 \mathrm{~T}$,

Nótese que en el caso de grupos de edad caracterizados por baja mortalidad, la aplicación directa del procedimiento de interpolación mencionado podría ocasionar el problema de que $\mathbf{2}^{\wedge}>1$. Por eso, en lugar de interpolar entre las proporciones de supervivencia $* \mathrm{~S}_{\mathrm{x}}$, es preferible interpolar entre las proporciones de mortalidad $1-$ ? , y así obtener el cociente de mortalidad $\mathrm{q}^{\wedge} \mathrm{y}$, entonces, derivar la probabilidad de retención $\mathrm{p}_{x}$ como $1 * \mathrm{q}_{x}-2$ ipi

$$
\mathrm{j} * \mathrm{i}
$$

En la práctica, sin embargo, en el caso de migración interregional, no es posible calcular las proporciones de supervivencia relativas a un período reciente, dado que los datos censales no proporcionan estimadores de las poblaciones involucradas al principio del período de observación. Este es, por ejemplo, el caso de los datos censales de migración en los Estados Unidos que contienen cambios de residencia en períodos quinquenales (por ejemplo, 1965-1970), pero no se puede hacer referencia a las poblaciones del principio del período (1965), ya que se desconocen. En tales circunstancias, se puede modificar el método de interpolación, primero, estimando un conjunto de probabilidades de transición $\mathrm{p}_{\mathrm{x}}$ condicionadas a la supervivencia $\mathrm{y}$, después, transformándolas en las probabilidades requeridas de transición, por introducir la información de mortalidad independiente. Entonces, $\mathrm{p}_{x}$ se obtiene de

$$
\mathrm{p}_{x}=\overline{\mathrm{p}}_{x} \hat{\mathrm{p}}_{x}^{\delta}
$$


donde $p_{x}$ es la correspondiente matriz de probabilidades de transición condicionada a la supervivencia y $\mathrm{p}^{\wedge}$ es la matriz diagonal cuyos elementos son similares a las probabilidades de supervivencia de las tablas ordinarias de vida. (Este procedimiento supone que las tasas de mortalidad dependen del lugar de residencia a la edad $x$, en vez del lugar de ocurrencia.) El procedimiento de interpolación permite calcular las diversas probabilidades de migración* $p_{x}(j \wedge i)$ condicionadas a la supervivencia; las probabilidades de retención condicionadas a supervivencia se obtienen, inmediatamente, de ${ }^{i} \mathbf{p}_{\mathrm{x}}=1-2 * \mathbf{P}_{\mathrm{x}}-$

Nótese que la exactitud de este procedimiento de interpolación para calcular las probabilidades de migración, condicionadas a la supervivencia o no, depende enormemente del tipo de fenómenos de movilidad que se tengan disponibles. Evidentemente, si el patrón de movilidad involucrado muestra fuertes variaciones por edad, el procedimiento de interpolación puede mejorar, si es que en algo, el método de promedio aritmético propuesto por Rees y Wilson (1977).

En la mayoría de los casos, sin embargo, los patrones de movilidad muestran regularidades muy estables. Entonces, es posible mejorar el procedimiento de interpolación, usando simultáneamente una graduación del típico patrón de movilidad. Por ejemplo, en el caso de migración interregional, dichas regularidades específicas por edad han sido comprobadas en el espacio (Long, 1973) y en el tiempo (Rogers y Castro 1976). Esto ha llevado recientemente al conocimiento de los patrones modelo de migración, similares a los patrones modelo de fecundidad (Rogers et. al, 1978). Entonces, se pueden mejorar enormemente los estimadores de las probabilidades de migración, especialmente aquéllas condicionadas a la supervivencia, ajustando un patrón modelo de migración, para cada par de regiones de origen y destino, al conjunto de proporciones de supervivencia.

La estimación de $\mathrm{p}_{\mathrm{x}}$ es relativamente directa y no se discute aquí.

Lo interesante de este método mejorado es que, en contraste con los métodos utilizados para estimar $p_{x}$ en el enfoque de movimiento, evita problemas empíricos tales como la ocurrencia de probabilidades negativas de retención. De hecho,

a) Los valores de las diversas probabilidades de transición ${ }^{*} p_{x}$, condicionadas a la supervivencia, están restringidos a estar entre cero y uno, como consecuencia del método de interpolación;

b) las probabilidades de supervivencia 'p£ se obtienen de la misma manera que las probabilidades de supervivencia de una tabla ordinaria de vida — son entre cero y uno, si se escoge el supuesto exponencial, y pueden ser negativas, si $\mathrm{n}$ es grande $\mathrm{y} / \mathrm{o} * \mathrm{M}_{\mathrm{x}}$ es grande ( $\sin$ embargo, es fácil salir de ese problema, consolidando las probabilidades de las tasas de mortalidad para grupos de edades individuales).

Ahora, ¿cómo se completa el cálculo de la tabla de vida de incrementos-decrementos basado en el enfoque de transición, una vez que se han observado las posibilidades de transición? El problema aquí es calcular adecuadamente $\mathrm{L}_{x}$, ya que este cálculo permite inmediatamente estimar el resto de las fracciones de la tabla de vida de estados múltiples, de las ecuaciones (41), (44) y (62). Como método alternativo al de integración lineal (Rogers, 1973; 1975), Ledent (1980b) sugirió un método más práctico, con el cual, mientras más grande sea 
la longitud, n, de los intervalos de edad, mejorará más con respecto al método de integración lineal.

\subsection{Enfoques de movimiento y transición: más contrastes}

En resumen, existen dos enfoques alternativos para la construcción de tablas de vida de incrementos-decrementos, dependiendo del tipo de datos disponibles. Uno, utiliza los datos en forma de movimientos registrados a lo largo de un intervalo dado de tiempo (enfoque de movimiento) y transformándolos en tasas de ocurrencia/exposición; el otro (enfoque de transición), usa datos en forma de cambios en los estados de presencia o residencia de los individuos, entre dos puntos en el tiempo, transformándolos en proporciones de supervivencia.

Como se mostró en la sección 3.2, el enfoque de movimiento lleva a fórmulas que expresan probabilidades de supervivencia específicas por edad en términos de las tasas de movilidad correspondientes, que no son características de una matriz propia de probabilidades de transición: en el caso en que el intervalo típico de edad, $n$, es grande, altas propensiones a migrar pueden llevar a probabilidades de retención numéricamente negativas, independientemente de si escogemos los métodos de integración exponencial o lineal.

En contraste, este problema afecta sólo ligeramente a la implementación del enfoque de transición, propuesta en la sección 3.3. La matriz de probabilidades de transición es el producto de una matriz de probabilidades de transición condicionadas a la supervivencia —que no es factible que sea impropia- y una matriz de proporciones de supervivencia -cuyos elementos diferentes de cero se calculan como en la tabla ordinaria de vida.

Este resultado, que el enfoque de transición es menos propenso a tener problemas durante su implementación, que el de movimiento, no es sorprendente, si se recuerda que cualquier tabla de vida es básicamente un modelo de transición. En efecto, el propósito principal de la tabla de vida es el de estimar probabilidades de transición, a partir de movimientos reales. En la tabla ordinaria de vida, esto no es problema: dado que la muerte no es un evento renovable, la consolidación de movimientos (es decir, muertes) en transiciones no es ambigua. En el caso de estados múltiples, sin embargo, dado que la movilidad es un evento renovable, dicha consolidación es menos directa y se puede concebir de dos maneras: ya sea que dicha consolidación se lleve a cabo con la ayuda de un proceso subyacente de movilidad o se haga al mismo nivel de los datos, para poder obtener datos equivalentes expresados en términos de transiciones (es decir, cambios en el estado de presencia).

Relacionando esto con los aspectos de los enfoques de movimiento y transición, lleva a concluir que,

a) en el primero, la consolidación de movimientos en transiciones está involu-

crada dentro del modelo que subyace a una tabla de vida de incrementos-de-

crementos: es precisamente donde entra el supuesto markoviano -o más exactamente, su equivalente discreto $Q m^{\wedge}$ independiente del estado de localización de la población estacionaria de estados múltiples)-;

b) en el segundo, la consolidación ocurre fuera del modelo.

Dejando a un lado las consideraciones empíricas — tales como aquellas que se relacionan con el uso de un método propio de estimación para calcular las proba- 
bilidades de transición, $\mathrm{p}_{\mathrm{x}}$ - ¿qué podemos inferir de tal conclusión con respecto a la esencia de los enfoques?

Evidentemente, si se pudieran aplicar los dos enfoques a situaciones idénticas de movilidad, se obtendrían diferentes resultados. El enfoque de transición, debido a su construcción, lleva a probabilidades de transición más o menos equiparables a las observadas. En contraste, el de movimiento no lleva a las mismas probabilidades de transición observadas, especialmente si el intervalo de edad, $\mathrm{n}$, es grande: en efecto, la consolidación de movimientos en transiciones dentro del modelo supone homogeneidad en las poblaciones e independencia vis-á-vis el pasado y, entonces, el enfoque de movimiento puede no ser apropiado para movimientos múltiples y migraciones de retorno dentro del intervalo unitario de tiempo. La validez de dicha declaración es sugerida en el trabajo de Singer y Spilerman $(1976,1978) \mathrm{y}$, en menor grado, por Rees (1977); y se puede demostrar por la comparación — para cada par de estados i y j- del número de movimientos por persona, calculado del modelo con la correspondiente razón observada. Entonces, el enfoque de movimiento generalmente lleva a matrices de probabilidades de transición con elementos fuera de la diagonal sobreestimados.

La conclusión aquí es que para una selección dada de n, es preferible el enfoque de transición que es el movimiento, ya que atenúa las fuertes consecuencias de la homogeneidad de la población y los supuestos markovianos.

Como la posibilidad de movimientos múltiples dentro de un intervalo dado aumenta con la longitud, $\mathrm{n}$, de dicho intervalo, mientras más grande sea el intervalo $\mathrm{n}$, mejor será la sobreestimación de los elementos de $\mathrm{p}_{\mathrm{x}}$ fuera de la diagonal, obtenida con el enfoque de movimiento; es decir, el enfoque de transición es preferible al de movimiento.

En la práctica, como los datos disponibles vienen ya sea en forma de movimientos o de transiciones, el problema principal no es el de escoger entre los dos enfoques alternativos, sino escoger el valor óptimo de $\mathrm{n}$ para la implementación de cada enfoque. Entonces, supongamos que podemos calcular una matriz quinquenal $\mathrm{p}_{\mathrm{x}}$ de probabilidades de transición -de ambas perspectivas, de movimiento y de transición- de dos maneras distintas:

a) directamente de los datos para grupos quinquenales de edad

b) del producto de matrices para edades individuales.

En el caso del enfoque de movimiento, no se espera que los resultados sean muy diferentes, dado que ambos métodos adoptan implícitamente la homogeneidad de la población y los supuestos markovianos. Entonces, como en la estimación de $\mathrm{p}_{x}$ surgen menos problemas empíricos cuando $\mathrm{n}$ disminuye, el mejor cálculo de una tabla de vida de incrementos-de crementos se obtiene cuando $\mathrm{n}$ es pequeño, es decir, cuando los datos vienen en grupos de edades individuales.

Por el contrario, en el de transición es más factible que el segundo método exhiba elementos fuera de la diagonal sobreestimados, que el primero, porque no es apropiado para movimientos múltiples y migraciones de retorno (Singer y Spilerman, 1976, 1978). Entonces, se concluye que con este enfoque se obtiene el mejor cálculo de una tabla de vida de incrementos-decrementos cuando $\mathrm{n}$ es grande. (Una n mayor da mejores estimados, no sólo de probabilidades de transición, sino también de años-persona vividos, L, si se utiliza el método original 
descrito en la sección 3.2.) En la práctica, parece ser óptimo que n tenga un valor de alrededor de 5 .

\section{CONCLUSIÓN}

La generalización de los conceptos de la tabla ordinaria de vida al caso de incrementos-decrementos es un asunto relativamente directo, en el que el álgebra de matrices se usa como un mecanismo de cálculo. Las diversas combinaciones de movimientos entre estados alternativos están representadas por fórmulas tan simples como las relativas a la tabla ordinaria de vida, siendo la única diferencia que los vectores y/o matrices reemplazan a los escalares. En el caso de las funciones de la tabla de vida, asociadas con grupos de individuos a una edad dada, existe una correspondencia directa entre las de las tablas ordinarias de vida y las de las tablas de incrementos-decrementos; así, se puede pasar de la primera a la segunda, con la sustitución apropiada de matrices por escalares. En contraste, en el caso de las funciones de la tabla de vida, asociadas con grupos de individuos en una categoría de edad dada (tasas de movilidad y proporciones de supervivencia), no hay tal correspondencia directa, ya que estas funciones dependen del estado de localización de la cohorte inicial.

Desde un punto de vista práctico, el elemento clave es la estimación de las probabilidades de supervivencia específicas por edad, de las que se origina el cálculo de todas las funciones de las tablas de vida de estados múltiples. Existen dos enfoques alternativos para tal estimación. Uno, consiste en duplicar la metodología usada comúnmente para hacer una tabla ordinaria de vida; esto se conoce como enfoque de movimiento, que considera a los pasajes entre estados como eventos instantáneos y, entonces, depende de entradas en forma de tasas de ocurrencia/exposición. Un aspecto importante de esta metodología es que necesita un supuesto adicional con respecto al modelo teórico, principalmente la independencia de las tasas de movilidad específicas por edad con respecto al estado de localización de la población estacionaria de estados múltiples. El otro, está relacionado con la construcción de la tabla ordinaria de vida, a partir de proporciones de supervivencia: esto se conoce como enfoque de transición y está dirigido a los estados de presencia de los individuos entre dos puntos en el tiempo. La implementación de este enfoque, como lo propuso Ledent (1980b), se dirige al cálculo de probabilidades específicas por edad, por interpolación entre proporciones de supervivencia observadas condicionadas a la supervivencia.

En la práctica, con la aplicación del enfoque de movimiento surgen algunos problemas empíricos:

¿z/la posibilidad de probabilidades negativas, especialmente si n es grande;

b) resultados inexactos que resultan de los supuestos subyacentes, markovianos y de homogeneidad de la población.

En contraste, el enfoque de transición es mucho menos afectado por tales problemas, especialmente cuando se implementa considerando que $\mathrm{n}$ tiene un valor de alrededor de 5 años.

La conclusión aquí es que, ceteris paribus, la construcción de una tabla de vida de incrementos-de crementos debe ser hecha, de preferencia, por el enfoque de transición y no por el de movimiento. Pero como los datos de movilidad gene- 
raímente vienen en forma de movimientos (tasas de ocurrencia/exposición) o (no y) transiciones (proporciones de supervivencia), el enfoque que se debe usar lo determina la forma en que hayan sido recolectados los datos.

\section{BIBLIOGRAFIA}

Chiang, C. 1960. "A stochastic study of the life table and its applications: II . Sample variance of the observed expectation of life and other biométrie functions". Human Biology, 32:221-238.

Cox, D. A.; Miller, H. D., 1965. The Theory of Stochastic Processes (John Wiley, New York).

Depoid, P., 1938. "Tables nouvelles relatives à la population française". Bulletin de la Statistique Générale de la France, 27:269-324.

Gantmacher, F. R., 1959. The Theory of Matrices 2 volumes (Chelsea. New York).

Hoem, J. M., 1970a. "A probabilistic approach to nuptiality". Biometrie-Praximetrie, 11:3-19.

Hoem, J. M., 1970b. "Probabilistic fertility models of the life table type". Theoretical Population Biology 1:12-38.

Hoem, J. M.; Fong, M. S., 1976. "A Markov chain model of working life tables". WP-2, Laboratory of Actuarial Mathematics, University of Copenhagen, Copenhagen.

Jordan, C. W., 1967. Life Contingencies (The Society of Actuaries, Chicago).

Keyfitz, N., 1968. Introduction to the Mathematics of Population (AddisonWesley, Reading, Mass.).

Krishnamoorthy, S., 1979. "Classical approach to increment-decrement life tables: an application to the study of the marital status of United States females, 1970". Mathematical Biosciences, 44:139-154.

Ledent, J., 1978. "Some methodological and empirical considerations in the construction of increment-decrement life tables". RM-78-25, International Institute for Applied Systems Analysis, Laxenburg, Austria.

Ledent, J., 1980a. "Constructing multiregional life tables using place-of-birthspecific migration data". WP-80-00, International Institute for Applied Systems Analysis, Laxenburg, Austria (forthcoming).

Ledent, J., 1980b. An improved method for constructing increment-decrement life tables from the transition perspective". WP-80-00, International Institute for Applied Systems Analysis, Laxenburg, Austria (forthcoming).

Long, L. H., 1973. "New estimates of migration expectancy in the United States". Journal of the American Statistical Association, 68 :(341 ) 37-43.

Long, L. H.; Hansen, K. A., 1975. "Trends in return migration to the South". Demography, 12:601-614.

Manninen, R., 1979. "Soumen vaestôn kehitysanalyysi neljàn âlueen jârjestelmânâ". (An analysis of the Finnish population as a system of four regions), Department of Geography, University of Helsinki, Helsinki.

McNeil, D. R.; Trusselll, T. J.; Turner, J. C, 1977. Spline interpolation of demographic data". Demography, 14:245-252.

Mertens, W., 1965. "Methodological aspects of the construction of nuptiality tables". Demography, 2: 317-348.

Rees, P. H., 1977. "The measurement of migration, from census data and other sources". Environment and Planning A, 9:247-272. 
Rees, P. H.; Wilson, A. G., 1977. Spatial Population Analysis (Edward Arnold. London)

Rogers, A., 1973. "The multiregional life $t^{\wedge} b l s^{\prime} . J o u r n a l$ of Mathematical Socio$\log y_{f} 2: 121-131$.

Rogers, A., 1975. Introduction to Multiregional Mathematical Demography (John Wiley, New York).

Rogers, A.; Castro, L. J., 1976. "Model multiregional life tables and stable populations". RR-76-9, International Institute for Applied Systems Analysis, Laxenburg, Austria.

Rogers, A.; Ledent, J., 1975. "Multiregional population projection". DP-128, Center for Mathematical Studies in Economics and Management Science, Northwater University, Evanston, III.; published in revised form in Cea, J. (Ed.), 1976. Optimization Techniquest: Modeling and Optimization in the Service of Man Part 1 (Springer, Berlin), pp. 31-58.

Rogers, A.; Ledent, J., 1976. "Increment-decrement life tables: a comment", Demography, 13:287-290.

Rogers, A.; Raquillet, R.; Castro, L. J., 1978. "Model migration schedules and their applications". Environment and Planning A, 10:475-502; also available as RM-77-57, International Institute for Applied Systems Analysis, Laxenburg, Austria.

Rogers, A.; von Rabenau, B., 1971. "Estimation of interregional migration streams from place of birth by residence data". Demography, 8:185-194.

Schoen, R., 1975. "Constructing increment-decrement life tables". Demography, 12:313-324.

Shoen, R.; Land, K. C, 1976. "Finding probabilities in increment-decrement life tables: a Markov process interpretation"..WP-7603, Program in Applied Social Statistics (PASS), Department of Sociology, University of Illinois at UrbanaChampaing, Urbana, III.

Schoen, R.; Land, K. C, 1977. "A general algorthm in estimating a Markovgenerated increment-decrement life table with applications to marital status patterns". WP-7715. Program in Applied Social Statistics (PASS), Department of Sociology, University of Illinois at Urbana-Champaign, Urbana, I I . ; forthcoming in Journal of the American Statistical Association, 1980.

Schoen, R.; Nelson, V. E., 1974. "Marriage, divorce, and mortality: a life table analysis". Demography, 11:267-290.

Singer, B.; Spilerman, S., 1976. "Some methodological issues in the analysis of longitudinal surveys". The Annuals of Economic and Social Measurement, S-A41-414.

Singer, B.; Spilerman, S., 1978. "Clusterning on the main diagonal in mobility matrices" in Sociological Methodology, 1979. Ed. K. Schuessler (Jossey-Bass, San Francisco). pp.261-296.

United Nations. 1961. Methods of Estimating Basic Demographic from Incomplete Data (United Nations, New York).

Willekens, F. J., 1978. "The demography of labor force participation". RM-7817, International Institute for Applied Systems Analysis, Laxenburg, Austria. 